\title{
Drosophila Conditioned Courtship: Two Ways of Testing Memory
}

\author{
Nikolai G. Kamyshev, ${ }^{1}$ Konstantin G. Iliadi, and Julia V. Bragina \\ Pavlov Institute of Physiology \\ St. Petersburg 199034, Russia
}

\begin{abstract}
In Drosophila, courtship reduction in male flies that have previous experience of courting a mated female is a result of the counterconditioning of an attractive unconditioned stimulus (US)- the aphrodisiac-which becomes an aversive conditioned stimulus (CS) after being paired with an aversive US - the antiaphrodisiac. In a retention test with a virgin female lacking the antiaphrodisiac, males retain a lower level of courtship for $3 \mathrm{hr}$ after training. However, a measure of courtship suppression, the learning index (LI), decreases significantly after only $1 \mathrm{hr}$. In contrast, in the retraining test with a mated female, the LI shows no decrease for $8 \mathrm{hr}$ but falls below significance $16 \mathrm{hr}$ after training. These results are discussed in terms of the transfer of training. Nonspecific transfer and nonassociative behavioral modifications play little, if any, role in the transfer of training. The retraining test is recommended as a new protocol for studying conditioned courtship. According to the model proposed here, in tests with a virgin female, the duration of memory retention is limited by the retention of the direct association between the $\mathrm{CS}$ and the aversive motivational system or by the retention of an internal representation of the US. In retraining tests, the CS-US association seems to be the only factor involved in transfer 3 or more hours after training.
\end{abstract}

\section{Introduction}

Behavior genetic investigations of memory in Drosophila often study the ability of flies to retain

${ }^{1}$ Corresponding author. memory for various times after conditioning. The longest memory retention has been reported for the classical conditioning procedure developed for Drosophila by Tully and Quinn (1985), elaborated from the original procedure of Quinn et al. (1974). The data obtained in this paradigm have led to important advances in our understanding of the process of memory formation in Drosophila (for review, see DeZazzo and Tully 1995). However, the main disadvantage of this procedure of Pavlovian discrimination is that it involves expensive and complex equipment. In addition, it requires large numbers of flies $(\sim 100$ flies to get one learning index value; this has to be replicated 5-10 times for statistical reasons). This tends to hamper experimental procedures such as injecting pharmacological agents.

Among other available classical conditioning procedures (for review, see Dudai 1988; Heisenberg 1989; Davis 1996), the conditioned courtship suppression paradigm (Siegel and Hall 1979) is the most attractive, because it is based on natural sexual behavior and involves only natural stimuli, requiring relatively small samples of flies, no complex equipment, and no special manipulation of the insects. This procedure can even be used for a single fly, thus providing an additional advantage (see, however, Materials and Methods).

Courtship in Drosopbila melanogaster is a very complex process, in which the two sexes exchange stimuli of different modalities: visual, chemosensory (olfactory and gustatory), and auditory (for review, see Hall 1994). Courtship-stimulating pheromones-the predominant cuticular hydrocarbons on the female cuticle-act at a very short distance (a few millimeters) and are perceived by contact (gustation) rather than by olfaction (for review of pheromonal studies, see Ferveur et al. 1989; Ferveur 1997).

Fertilized Drosophila melanogaster females stimulate males to court them (Bastock and Manning 1955), but this courtship is less vigorous than that elicited by virgins (Cook and Cook 1975; Sie-

LEARNING \& MEMORY 6:1-20 @ 1999 by Cold Spring Harbor Laboratory Press ISSN1072-0502/99 \$5.00

$$
\begin{array}{lllllllllllllllll}
L & E & A & R & N & I & N & G & \boldsymbol{Q} & M & E & M & O & R & Y \\
\mathbf{1} & & & & & & & & & & & & & & & &
\end{array}
$$


gel and Hall 1979). This change in the male's behavior coincides with a change in female's behavior: The fertilized female begins to extrude her ovipositor in response to male courtship, which blocks most copulation attempts (Connolly and Cook 1973). Mating also results in the appearance or increase of the antiaphrodisiac content in the pheromonal profile of fertilized females. Despite strong, though indirect (behavioral), evidence for its existence, identification of the natural antiaphrodisiac and the question of how mating triggers an increase of its content in a fertilized female has been a complex and controversial story (Ferveur et al. 1989; Ferveur 1997; see references in Hall 1994).

If a male courts a fertilized female he tends not to court subsequent females: for $2-3 \mathrm{hr}$ if he is then placed with a mature virgin female (Siegel and Hall 1979) and for nearly 1 day if he is then placed with a fertilized female (Gailey et al. 1984). A fertilized female with crushed head can modify male courtship behavior only if she has been courted by another male just before having her head crushed. This suggests that a necessary cue is released by a female in response to male courtship (Gailey et al. 1984). A "conditioning" hypothesis has been formulated to explain this phenomenon (Tompkins et al. 1983; Gailey et al. 1984), according to which courtship reduction results from the association of two chemical cues. When the male courts a fertilized female, he learns to associate courtship-stimulating and courtship-inhibiting cues, so that when subsequently presented with only courtship-stimulating cues (virgin female), his courtship response is repressed in "anticipation" of an aversive cue. This suggests that the aphrodisiac acts as a conditioned stimulus (CS), being reinforced by the antiaphrodisiac as an unconditioned stimulus (US). The conditioning hypothesis was supported by further studies (Ackerman and Siegel 1986; Zawistowski 1988). Zawistowski (1988) also suggested interpreting this kind of learning as counterconditioning of inhibitory and excitatory stimuli (see Mackintosh 1974), during which the excitatory stimulus (aphrodisiac) either acquires aversive qualities or loses its excitatory capacity after pairing with the inhibitory stimulus (antiaphrodisiac).

Conditioned courtship suppression is widely used to test learning ability in various kinds of mutants, strains, and transgenic flies (see references in van Swinderen and Hall 1995). Although it has been known that the reduction in courtship activity lasts longer if trained males are tested with fer- tilized females rather than with virgins (Gailey et al. 1984), no systematic studies of this phenomenon have been done. However, this possibility provides a valid way of testing memory in conditioned courtship experiments. Spear (1971) notes that the retention test not only assesses but also determines what is "remembered." Retrieval of a target memory attribute depends on the presence of certain cues at the time of the retention test, which activate other attributes of the memory. Some of the more important attributes for retrieval of a memory are those representing the conditions of reinforcement. Reproducing the conditions of reinforcement on subsequent relearning trials may activate a sufficient number of additional attributes to cause better memory performance than in a retention test without reinforcement. Accordingly, Spear (1971) distinguished two measures of retention failure, which we use here. He defined a "lapse" as an initial and transient failure to perform in accordance with the most recent learning, which is measured before re-exposure to the most recent reinforcement conditions. The lapse may be measured on the first trial following the retention interval or in terms of transfer to different reinforcement conditions such as extinction. The other measure, "loss,' is defined as a decrement in performance of the most recent learning given the reproduction of the most recent reinforcement conditions (e.g., a decrement in the rate of relearning).

Using a retraining test to measure memory performance will be considered here in terms of training transfer. A study of transfer is by definition a two-stage experiment comprising a training phase and a test phase. The basic question is how the training provided during the first stage influences performance during the second stage (Postman 1971). The presence of significant transfer effects implies that at least some of the responses established during the training phase are carried over to the test task. The effects are positive or negative depending on whether these responses are appropriate or inappropriate in the new situation. We have maximum positive transfer when the training and test tasks are identical. Although this situation is usually considered as a distributed (spaced) practice (Houston 1981), instead of focusing on the cumulative effects of practice it is possible to use the transfer test as an analytic device to determine what has been learned in the training phase (Postman 1971).

In this paper we compare two memory tests:

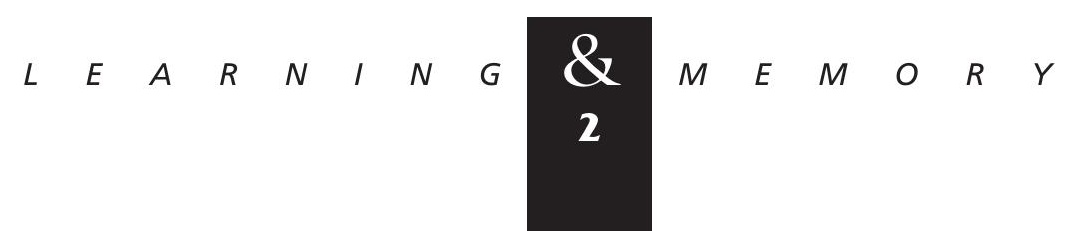


tests with virgin females (the retention test), which reveal memory retention, and tests with mobile fertilized females (the retraining test), which reveal the transfer of training. Although the two kinds of memory may differ, the measures of memory performance are similar and allow quantitative comparison.

We have shown that soon after training, memory performance is the same whether the test is performed with virgin or fertilized females. However, $1 \mathrm{hr}$ after training, memory performance in tests with virgins significantly decreases, demonstrating memory lapse. In contrast, in tests with mated females, trained males show no memory loss for at least $8 \mathrm{hr}$. A working model of conditioned courtship is suggested, which reveals the components of training transfer and explains the difference in the dynamics of memory performance observed in retention and retraining tests.

\section{Materials and Methods}

\section{DROSOPHILA STRAINS AND CULTURE CONDITIONS}

Flies were raised and kept on standard yeastraisin medium (15 grams of agar, 25 grams of dried yeast, 30 grams of sugar, 30 grams of semolina, 30 grams of raisins, with water added up to 1 liter of final volume) under $25^{\circ} \mathrm{C}$ and a $12: 12$ light-dark cycle. The wild-type outbred strain Canton-S (C-S) maintained in the laboratory for several decades was used in the experiments.

\section{BEHAVIORAL PROCEDURES}

Experimental males were collected without any anesthesia soon after eclosion and kept individually in culture vials with yeast-raisin medium for 5 days until the experiment was performed. All females used for training and testing were collected as virgins (10-12 flies per vial). A day before the experiment, 10-12 4-day-old females were brought together with 15-18 4-day-old males in one vial and left there for mating till the test, that is, for 18-22 hr. All matings, as a rule, occurred within 15-20 min. Experiments were performed at $25^{\circ} \mathrm{C}$; humidity was not controlled.

Male courtship behavior was observed in a perspex experimental chamber (15 mm diam., $5 \mathrm{~mm}$ high) with a movable transparent perspex cover, a sliding opaque partition that divided the chamber into two halves, and two lateral entries $(3 \mathrm{~mm}$ diam.) with stoppers. Before training or testing, each chamber was cleaned with 50\% ethanol and dried.
For training, a naive 5-day-old male (with no experience of sexual contact) was placed into an experimental chamber together with a 5-day-old mated $C-S$ female. The male and the female were introduced into the chamber by shaking through a funnel, through separate holes, with the divider in place. After several minutes to recover from the transfer, the divider was withdrawn, and the flies were left together for $30 \mathrm{~min}$.

Tests for memory performance were performed at various times after training: immediately after training; at 15, 30, and $60 \mathrm{~min}$; and at 3, 8, 16, and $24 \mathrm{hr}$. If the test was performed at $8 \mathrm{hr}$ or later, the experimental male was isolated in a vial with a medium; otherwise, he was left in the same chamber, whereas the female was isolated. The experimental male was moved from his chamber to the chamber with the test female through connecting holes, the divider in the testing chamber being in place. Isolating and moving the fly from chamber to chamber was performed cautiously, facilitated by the use of the divider and negative geotaxis. The aspiration technique was not used because it disturbs the flies. Experimental males were tested either with etherized virgin or mobile mated $C-S$ females, both 5 days old.

An ethogram of the male's behavior was recorded using a computer program written by the authors. Male courtship was observed for $300 \mathrm{sec}$. The onset of various courtship elements (orientation and pursuit, vibration, licking, attempted copulation; see Bastock and Manning 1955) and noncourtship behaviors (locomotion, preening, rest) was recorded by pressing a key on the computer keyboard. Recording began with the onset of the first courtship element, not later than 45-50 sec after placing the experimental male into the chamber. During this empirically determined period, many males do not pay attention to females but display exploratory behavior and preening probably induced by the mechanical disturbance. The time sequence for the behavioral elements specified was recorded for each experimental male. Another program was used for decoding the ethograms, calculating various behavioral parameters, and organizing the resulting data. In this study the total time spent in courtship is analyzed.

EXPERIMENTAL DESIGNS

AND STATISTICAL PROCESSING

GENERAL CONSIDERATIONS AND EXPERIMENTAL DESIGNS

Although in our experiments the total time of

$$
\begin{array}{llllllllllllllll}
L & E & A & R & N & I & N & G & \boldsymbol{\bigotimes} \\
3 & M & E & M & O & R & Y
\end{array}
$$


Kamyshev et al.

Table 1: Comparison of behavior of naive and sham control males in immediate tests with immobilized virgin and mobile mated females

\begin{tabular}{lllcccc}
\hline Test & Group & $N$ & $\mathrm{Cl}$ & $U$ & $U_{0.025}$ & $P$ \\
\hline Immobilized virgin females & naive & 20 & $41.9 \pm 6.66$ & & & \\
& sham control & 20 & $38.3 \pm 8.47$ & 221 & 127 & $>0.05$ \\
\multirow{2}{*}{ Mobile mated females } & naive & 20 & $45.0 \pm 5.87$ & & & \\
& sham control & 20 & $42.8 \pm 5.44$ & 207 & 127 & $>0.05$ \\
\hline
\end{tabular}

$\mathrm{Cls}(\mathrm{Cl} \pm$ S.E.M. $)$ calculated for independent samples of naive and sham control males are shown. Naive males were kept individually from the moment of eclosion. For sham training, a male was placed alone in an experimental chamber for 30 min. After sham training, flies were tested immediately either with immobilized virgin or mobile mated 5-day-old C-S females. $N$ denotes sample size. The Mann-Whitney U-statistic was calculated to compare groups of naive and sham control males. The null hypothesis was rejected when $U \leqslant U_{\alpha / 2}$ (two-tailed test). $P$ is the probability of the null hypothesis.

observing courtship behavior is fixed (300 sec), all the data are presented in terms of a courtship index (CI), that is, the percentage of time spent in courtship, with the aim of comparing them with the data of other investigators.

There are two ways of obtaining CI values for naive and trained males. The first is to estimate CIs in the same individual before and after training. The second is to examine independent samples of naive and trained males. The first approach is inadequate for measuring memory performance over a long time after training, because every uncontrollable factor that influences courtship activity and depends on time will contribute to differences in the CI. That is why we randomly tested individuals from independent samples of naive and trained males, in a blind experiment. In practice, when the memory performance was measured $0-3 \mathrm{hr}$ after training, the same sample of naive males was used as a control. Each of the more delayed tests $(8,16$, and $24 \mathrm{hr}$ after training) had its own control naive males tested simultaneously with a sample of trained males.

Unlike other investigators (e.g., Kane et al. 1997), we did not exclude males with a low courtship level from behavioral tests, because this pro- cedure may create differences in CI between naive and trained males in the absence of learning. ${ }^{1}$ Instead, the experiments were finished and all data discarded if the courtship activity of the first 3-5 naive males on the given day was low for some reason.

Comparison of behavior in the naive and sham control individuals (males placed alone in experimental chambers for $30 \mathrm{~min}$ ) shows no difference between the two experimental groups (Table 1). The sham control was not used in subsequent experiments.

To measure the transfer of training in the test with mated females, a widely used index of relative transfer (Postman 1971) was calculated as the percentage of improvement shown by the experimental group (previously trained males) over the control group (naive males):

$$
\text { percent transfer }=\frac{E-C}{C} \cdot 100
$$

where $E$ and $C$ are the scores of the experimental and control group, respectively. If the scores vary inversely with the level of performance (as in the case of CIs), the difference $C-E$ is used in the

\footnotetext{
${ }^{1}$ Let $A$ be a level of sexual arousal of a male in a population. Let us assume that in the absence of learning, $A$ is constant for each male but shows interindividual variability. Let us further assume that $A$ determines the level of courtship under various conditions (courting mated or virgin females, etc.), such that under any condition the greater the value of $A$, the greater the mathematical expectation of $\mathrm{Cl}$. Excluding males with a low initial level of courtship from behavioral tests should obviously truncate the left tail of the sample distribution of $A$. If the same procedure is not performed in the control group of naive males, this would result in a bias in the courtship level between experimental and control groups in the absence of learning. This bias has a direction, which is opposite to the changes in courtship level owing to conditioning, and hence may hide weak aftereffects of training (e.g., in the delayed tests). Such problems are present even when learning is measured in one individual. The $\mathrm{Cl}$ observed in any given male results, in fact, from time sampling. If all observations are truncated below some minimum level at the first time interval, but this is not performed at the next time interval, artifactual changes in $\mathrm{Cl}$ would be observed, coinciding in direction with changes expected from learning.
}

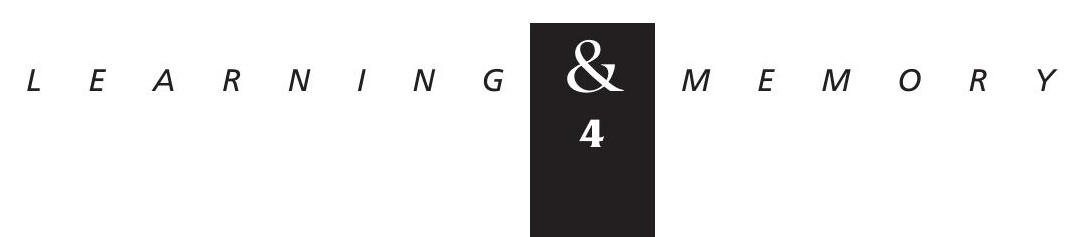


numerator. The final formula for relative transfer in our case is thus identical with the formula used by Gailey et al. (1982, 1984) for the learning index (LI) that is used to measure memory retention in the test with virgin females:

$$
\mathrm{LI}=\frac{\mathrm{CI}_{\mathrm{na}}-\mathrm{CI}_{\mathrm{tr}}}{\mathrm{CI}_{\mathrm{na}}} \cdot 100=\left(1-\frac{\mathrm{CI}_{\mathrm{tr}}}{\mathrm{CI}_{\mathrm{na}}}\right) \cdot 100
$$

where $\mathrm{CI}_{\text {na }}$ and $\mathrm{CI}_{\mathrm{tr}}$ are the mean courtship indices for independent samples of naive and trained males, respectively. An LI value of 0 corresponds to a complete absence of learning or full memory loss (lapse), whereas a value of 100 (complete suppression of courtship in trained males) is the maximum possible value for best learners. Using the LI has the following advantages over using the difference score $\mathrm{CI}_{\mathrm{na}}-\mathrm{CI}_{\mathrm{tr}}$ : (1) This relative measure allows comparison of experimental groups that may differ in the initial level of courtship in naive males, and (2) cases where males do not court at all (and, thus, receive no training) do not contribute to $\mathrm{LI}^{2}{ }^{2}$

We did not use the ANOVA, because only a small portion of comparisons that were of interest in this study could be correctly dealt with using this method. Three different statistical approaches to conditioned courtship data were applied and compared here. All statistical decisions were made at the significance level of $\alpha=0.05$. One-tailed testing against the null hypothesis was used in the following cases: (1) to compare naive versus trained males and for $H_{0}: \mathrm{LI}=0$, that is, to estimate the memory performance that was revealed by $\mathrm{CI}_{\mathrm{na}}>\mathrm{CI}_{\mathrm{tr}}$, whereas the reverse situation $\left(\mathrm{CI}_{\mathrm{na}}<\mathrm{CI}_{\mathrm{tr}}\right)$ had no relation to learning and (2) to compare the LIs at various times after training with initial $\mathrm{LI}\left(\mathrm{LI}_{0}\right)$ just after training, that is, to determine the beginning of memory loss (lapse) that was revealed by $\mathrm{LI}<\mathrm{LI}_{0}$. In all other cases, twotailed tests were used.

\section{AN APPROACH BASED ON NORMAL THEORY}

The approach used in a recent paper on conditioned courtship by van Swinderen and Hall
(1995) was used here. Briefly, the raw CIs were transformed into degrees (arcsine transformation, which is usually aimed at equalizing the sample variances in case of binomial distribution; Sokal and Rohlf 1995). The calculated LIs based on the transformed data were corrected by replacing the ratio $\mathrm{CI}_{\mathrm{tr}} / \mathrm{CI}_{\text {na }}$ in equation 2 with the corrected estimate (the reason for correction was that the $\mathrm{CI}_{\mathrm{tr}} / \mathrm{CI}_{\mathrm{na}}$ ratio was biased). This gave a formula for a corrected LI:

$$
\mathrm{LI}_{\text {cor }}=\left[1-\frac{\mathrm{CI}_{\mathrm{tr}}}{\mathrm{CI}_{\mathrm{na}}} \cdot\left(1+\frac{\operatorname{Var} \mathrm{CI}_{\mathrm{na}}}{\mathrm{CI}_{\text {na }}^{2}}\right)\right] \cdot 100
$$

To apply the Student $t$-test for comparing two LIs, the following estimation of the LI standard error was used:

$$
\text { S.E.M. }{ }_{\mathrm{LI}}=\frac{\mathrm{CI}_{\mathrm{tr}}^{2}}{\mathrm{CI}_{\mathrm{na}}^{2}} \cdot\left(\frac{\operatorname{Var} \mathrm{CI}_{\mathrm{tr}}}{\mathrm{CI}_{\mathrm{tr}}^{2}}+\frac{\operatorname{Var} \mathrm{CI}_{\mathrm{na}}}{\mathrm{CI}_{\mathrm{na}}^{2}}\right)
$$

A joint Student $t$-test (based on the assumption that variances are equal) was used for comparing both CI and LI.

\section{AN APPROACH BASED ON WILCOXON'S TEST}

CIs were compared by means of two distribution-free tests: the Wilcoxon $W$ and the Mann-Whitney $U$ (Hollander and Wolfe 1973). The $W$-statistic was used to estimate the nonparametric version of the LI (see below); the $U$-test was used because of the more complete tables that are available. In the absence of table values for the W-statistic, Iman's improved approximation $J$ for large samples was used (Iman 1976).

To construct the nonparametric version of the LI, all individual raw courtship indices were divided by the mean CI of control naive males and multiplied by 100 . After such a transformation, the mean CI of naive males was equal to 100 in every experimental group, which allowed us to use the

\footnotetext{
${ }^{2}$ This can easily be shown to be true by some simple algebraic calculations. Suppose, for the sake of simplicity, that in some hypothetical sample before training all $n$ males have the same level of courtship $b$ (the same level of courtship is expected for the independent sample of naive males, because both samples are derived from the same population) and after training this level is decreased to $a$. The mean difference between $\mathrm{Cl}_{n a}$ and $\mathrm{Cl}_{\mathrm{tr}}$, then, will be $d=n(b-a) / n=b-a$ and $\mathrm{LI}=1-a / b$. Let in another sample $m$ among $n$ males show no courtship at all, whereas others have the same level of courtship before and after training, as in the first sample. Then $d=(n-m)(b-a) / n=(1-m / n)(b-a)$ and $\mathrm{LI}=[(1-m / n)(b-a)] /[(1-m / n) b]=1-a / b$. Although the difference score depends on the presence of inactive males, those cases are automatically excluded when the $\mathrm{LI}$ is calculated. Thus, the only negative effect of the presence of noncourting males in a sample is a decrease in the precision of estimating the LI.
}

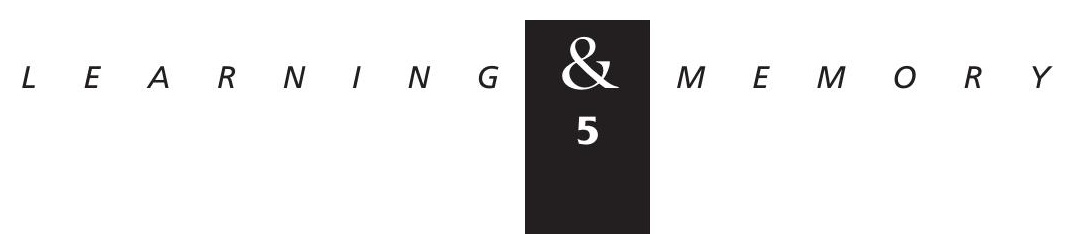


difference $\mathrm{CI}_{\text {na }}-\mathrm{CI}_{\mathrm{tr}}$, instead of LI (equation 2), as a relative measure of memory performance. This transformation (division by constant) did not change the ranks of individual CIs within each experimental group and, consequently, did not change the values of $W$ - and $U$-statistics.

The estimator $\Delta$ of Hodges and Lehmann (Hollander and Wolfe 1973) related to the $W$-statistic was applied to the transformed CIs and used as a point estimator for nonparametric LI $\left(\mathrm{LI}_{n p r}\right)$. If there are $n_{1}$ individuals in the sample of naive males and $n_{2}$ individuals in the sample of trained males, $n_{1} n_{2}$ possible $\mathrm{CI}_{\mathrm{na}}-\mathrm{CI}_{\mathrm{tr}}$ differences should be calculated. The median of this data vector is the value of $\mathrm{LI}_{\mathrm{npr}}$. Distribution-free confidence interval of Moses (Hollander and Wolfe 1973) based on the Wilcoxon $W$-test was used as a confidence interval for $\mathrm{LI}_{\mathrm{npr}}$.

\section{RANDOMIZATION TESTS}

The creator of this method was Fisher (1966). For basic principles see also Basu (1980) and Edgington (1980). It is also described in detail by Sokal and Rohlf (1995). Randomization analysis is based on looking through all possible hypothetical experimental plans and calculating the probability of getting the value of statistic of interest obtained in the real experiment by chance.

A set of hypothetical experimental plans is generated by permutations between samples. In case of two samples, for example, the algorithm is as follows. Two data sets, representing each sample, are joined into one data vector. Then, the latter is used to form two data sets (with the same size as real samples) by chance. In practical terms, a random number generator is used to define which elements from the joint data vector will belong to the first data set, the others belonging by default to the second. This procedure results in creating one random experimental plan. If it is conceivable to create all possible experimental plans, where each plan is unique, such a test is called an exact randomization test. When the number of possible experimental plans is too large, it is sufficient to generate a sample of random experimental plans. We have used this sampled randomization test (Sokal and Rohlf 1995) here. In all cases, the number of random experimental plans generated (M) was equal to 10,000 .

For each of the random plans, the statistic of interest $(S)$ was calculated and compared with that obtained in the real experiment $\left(S_{r}\right)$. The greater the number of cases for which $S \geqslant S_{r}$, the more probable it is that the result of a real experiment may be obtained by chance under the existing data variability, that is, the greater the probability of the null hypothesis. The number of hypothetical experiments $(K)$ promoting $H_{0}$, that is, fitting the condition $S \geqslant S_{r}$ (for the one-sided test) or $|S| \geqslant\left|S_{r}\right|$ (for the two-sided test), was counted. The minimum significance level, $\alpha_{R}$, at which the null hypothesis might be rejected, was determined as $\alpha_{\mathrm{R}}=K / M$. The null hypothesis was rejected if $\alpha_{\mathrm{R}} \leqslant 0.05$.

Randomization analysis was applied for solving the following tasks:

1. Comparison of two CIs. The statistic used was the difference between corresponding sample means.

2. Test of the significance of the LI. The statistic used was LI calculated by formula 2 .

3. Comparison of two LIs. The statistic used was the difference between two LIs. Permutations were performed here only between samples of naive and trained males within each experimental condition.

\section{Results}

\section{MEMORY PERFORMANCE IN THE TEST} WITH IMMOBILIZED VIRGIN FEMALES

We first sought to reproduce earlier findings (Siegel and Hall 1979; Gailey et al. 1984). Males that had courted mated females for 30 min were tested for $5 \mathrm{~min}$ with immobilized virgin females at various times after training. The time spent courting (CI) is minimal just after training. It then increases continuously over $3 \mathrm{hr}$ with an exception at $15 \mathrm{~min}$, when the $\mathrm{CI}$ is temporarily raised and then returns to a lower value at $30 \mathrm{~min}$ after training (Table 2A). Several estimates are used here as measures of memory performance. The first is the difference between the CIs of naive and trained males. Its significance is estimated by both parametric and nonparametric methods. The one-tailed $t$-test (see Materials and Methods for the rationales of using one-tailed tests in this study) shows that this difference is significant at $\alpha=0.05$ for the whole of the 3-hr period. However, the joint $t$-test used is valid only when the variances are equal (Sokal and Rohlf 1995). For raw data (Table 2A), this is true only for the final 3 -hr point (see $F$-ratio). The arcsine transformation (Table 2B) that is normally used to equalize variances in case of binomial

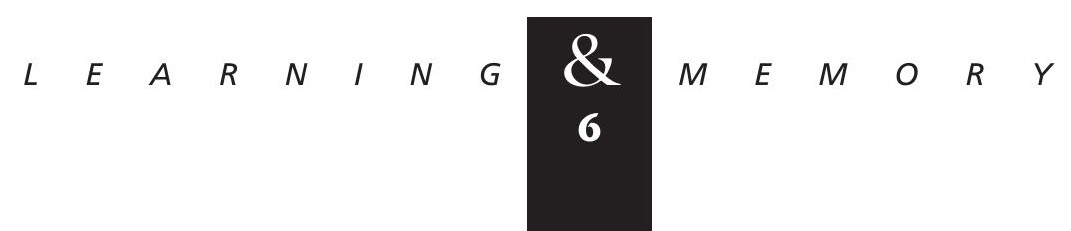


Table 2: Dynamics of memory performance in the tests with virgin female

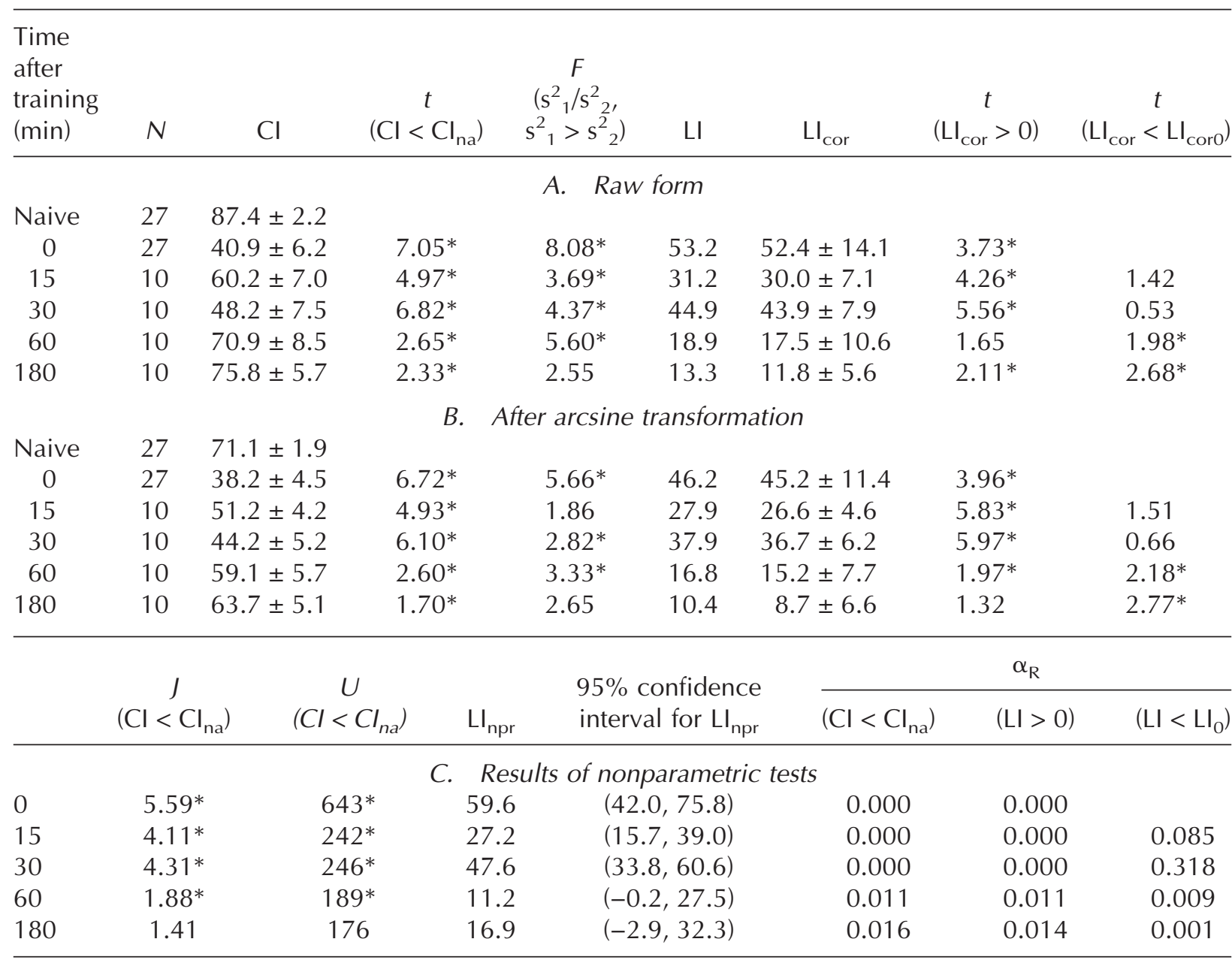

Cls $(\mathrm{Cl} \pm$ S.E.M.) calculated for independent samples of naive and trained males ( $N$ denotes sample size) at different times after 30 min of training with mated females are presented either in their raw form $(A)$ or after an arcsine transformation $(B)$. The values of $t$ for the difference in $\mathrm{Cl}$ between naive and trained males $\left(\mathrm{Cl}_{\mathrm{na}}-\mathrm{Cl}_{\mathrm{tr}}\right)$ are presented in the fourth column. ${ }^{(*)}$ Cases when the one-tailed joint $t$-test rejects $H_{0}$ against the alternative hypothesis $\mathrm{H}_{1}: \mathrm{Cl}_{<} \mathrm{Cl}$ na (i.e., the $\mathrm{Cl}$ at a given time after training is less than the $\mathrm{Cl}$ of naive males) at $\alpha=0.05$. In the fifth column, the corresponding variance ratio (a greater sample variance divided by a smaller sample variance) is presented; F-ratios significant at $\alpha=0.05$ are labeled, showing cases when the $t$-test should be considered as incorrect. The LI was calculated for both raw and transformed data according to Gailey et al. $(1982,1984)(\mathrm{LI})$ and with a bias correction ( $\mathrm{LI}_{\text {cor }}$ ) according to van Swinderen and Hall (1995). The corresponding formulas are given in Materials and Methods. Standard errors were calculated and $t$-tests were performed as in van Swinderen and Hall (1995) for $\mathrm{LI}_{\text {cor }}$. In the eigth column, the results of a one-tailed $t$-test for $H_{0}$ : $\mathrm{LI}_{\text {cor }}=0$ against $H_{1}: \mathrm{LI}_{\text {cor }}>0$ are presented. In the ninth column, to detect the beginning of memory lapse, the null-hypothesis that $\mathrm{LI}_{\text {cor }}$ at a given time after training does not differ from its initial value after training is tested against the alternative hypothesis that the current $\mathrm{LI}$ is less than the initial index. $C$ shows the results of nonparametric tests. The alternative hypothesis, against which $H_{0}$ is tested, is denoted in parentheses. $J$ is the Iman's approximation for large samples for the Wilcoxon $W$-statistic, $U$ is the Mann-Whitney statistic, $\mathrm{LI}_{\mathrm{npr}}$ is the Hodges-Lehmann estimator applied to individual Cls divided by the mean $\mathrm{Cl}$ of naive males (details in Materials and Methods). Ninety-five percent confidence intervals for $\mathrm{LI}_{\mathrm{npr}}$ are presented in the fifth column. All statistical comparisons resulting in the rejection of null hypotheses at $\alpha=0.05$ are indicated with asterisks. The final columns of $C$ present the results of the randomization tests, each based on 10,000 permutations (see Materials and Methods). $\alpha_{R}$ is the directly computed probability of rejecting the null hypothesis (the alternative hypothesis is indicated in parentheses).

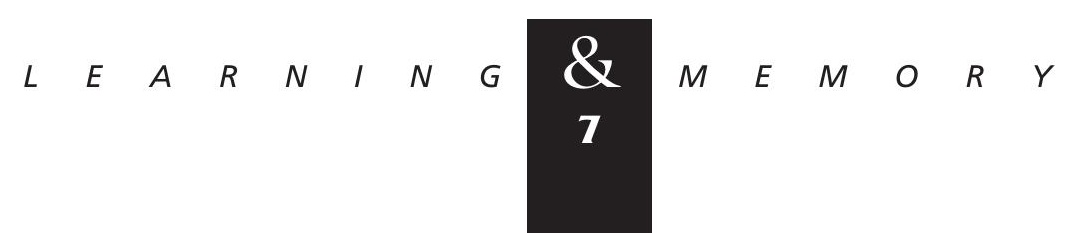


data distribution leads to only a slight decrease in the $F$-ratio, leaving it significant at most time points. What is important, however, is that $t$-test is valid both for raw and transformed data at the 3-hr point and shows significant memory retention at this time. Nonparametric, distribution-free Wilcoxon and Mann-Whitney tests show significant memory retention for all time points except the last one (Table 2C). This is in accordance with the usual lower efficiency of rank statistics as compared with those based on interval scales (Runyon 1977). The randomization test, which is distribution free but is based on the interval scale, gives the same result as the $t$-test, revealing significant memory retention for all time points.

Another measure used for estimating memory retention is the LI. This was calculated for both raw and transformed data according to Gailey et al. (1982, 1984) as LI (equation 2) and according to van Swinderen and Hall (1995) as $\mathrm{LI}_{\text {cor }}$ (equation 3), that is, LI corrected for bias (see Materials and Methods). Standard errors were calculated and $t$ tests applied only for $\mathrm{LI}_{\text {cor }}$ according to van Swinderen and Hall (1995). LI based on transformed data is lower than LI based on raw data. The correction for bias produces only a slight effect. Values of the $t$-statistic calculated for $H_{0}: \mathrm{LI}_{\text {cor }}=0$ against $H_{1}: \mathrm{LI}_{\mathrm{cor}}>{ }_{\text {cor }} \mathrm{O}$ are quite different from $t$ values calculated for the difference in CI between naive and trained males. Although the difference in CI is significant for all time points in the case of both raw and transformed data (Table $2 \mathrm{~A}, \mathrm{~B}$ ), $\mathrm{LI}_{\text {cor }}$ shows no significant difference from zero at the 1-hr point in the case of raw data (Table $2 \mathrm{~A}$ ) and at the 3-hr point in the case of transformed data (Table 2B). In contrast to the $t$-test, the randomization analysis shows very close values of $\alpha_{R}$ (Table 2C) for these two null hypotheses $\left(\mathrm{CI}=\mathrm{CI}_{\mathrm{na}}\right.$ and $\mathrm{LI}_{\text {cor }}=0$ ). This is expected, because they are closely related. Both null hypotheses were rejected for all time points. We also used the nonparametric variant of the $\mathrm{LI}, \mathrm{LI}_{\mathrm{npr}}$ (see Materials and Methods), based on the Hodges-Lehmann estimator related to the $W$-statistic of Wilcoxon (Table 2C). This estimator is less sensitive to rough errors than its analog (sample mean) from the normal theory (Hollander and Wolfe 1973). $\mathrm{LI}_{\mathrm{npr}}$ gives values rather close to the values of LI. The 95\% confidence interval for $\mathrm{LI}_{\mathrm{npr}}$ (see Materials and Methods) includes zero at the two last time points. This disagreement with the one-tailed $W$-test arises because the confidence interval is based on the twotailed $W$-test. The absence of an overlap of confi- dence intervals for $L_{\text {npr }}$ shows that memory performance 15,60 , and $180 \mathrm{~min}$ after training is significantly less than just after training. Both $t$ - and randomization tests reveal only the last two differences.

Taken together, the data presented in Table 2 mean that, when tested with virgins, males retain a lower level of courtship activity for $\sim 3 \mathrm{hr}$. However, the extent of conditioned suppression (memory performance revealed by LI) decreases significantly $1 \mathrm{hr}$ after training. A reversible reduction in LI seems to take place $15 \mathrm{~min}$ after training completion.

\section{MEMORY PERFORMANCE IN THE TEST WITH MOBILE MATED FEMALES}

The data obtained in tests with mobile mated females are presented in Table 3 . The arcsine transformation equalizes the variances of naive and trained males at all time points making the $t$-test valid for the transformed data (Table 3, cf. F-ratio in A and B). All statistics used $\left(t, J, \alpha_{\mathrm{R}}\right)$ show that the $\mathrm{CI}$ of trained males remains significantly lower than the CI of naive males at least for $8 \mathrm{hr}$, whereas 16 $\mathrm{hr}$ after training this difference is no longer significant. The same conclusion follows from consideration of the three kinds of LI index: LI (see $\alpha_{\mathrm{R}}$ for $H_{1}$ : LI > 0; Table 3C), $\mathrm{LI}_{\text {cor }}$ (see corresponding $t$ statistics) and $\mathrm{LI}_{\mathrm{npr}}$ (see 95\% confidence interval in Table 3C). Whereas values of $\alpha_{\mathrm{R}}$ for $H_{1}$ : CI $<\mathrm{CI}_{\text {na }}$ and $H_{1}: \mathrm{LI}>0$ are very similar, the agreement between the corresponding $t$-statistics is not as close. Moreover, although values of $\mathrm{LI}$ and $\mathrm{LI}_{\mathrm{npr}}$ are very close and positive for all time points, values of $\mathrm{LI}_{\text {cor }}$ are negative 16 and $24 \mathrm{hr}$ after training. "Bias correction"- - used for calculating $\mathrm{LI}_{\text {cor }}$-leads to unreliable data, when $\mathrm{LI}_{\text {cor }}<0$, whereas $\mathrm{CI}_{\mathrm{tr}}<\mathrm{CI}_{\text {na }}$. The $t$-test, based on $\mathrm{LI}_{\text {cor }}$, and the randomization test, based on LI, do however show similar results when LIs at various times after training are compared with the initial LI just after training $\left(H_{0}\right.$ : $\mathrm{LI}=\mathrm{LI}_{0}$ against $H_{1}: \mathrm{LI}<\mathrm{LI}_{0}$ ). Initial memory performance is retained for at least $8 \mathrm{hr}$ but decreases severely $16 \mathrm{hr}$ after training. Among the $95 \%$ confidence intervals for $\mathrm{LI}_{\mathrm{npr}}$, only that for $24 \mathrm{hr}$ after training has no overlap with the initial one.

\section{COMPARISON OF MEMORY PERFORMANCE IN RETENTION AND RETRAINING TESTS}

Memory performance is significantly better in the test with mated females 15, 60, and $180 \mathrm{~min}$ after training (Table 4). This is indicated by $t$-test

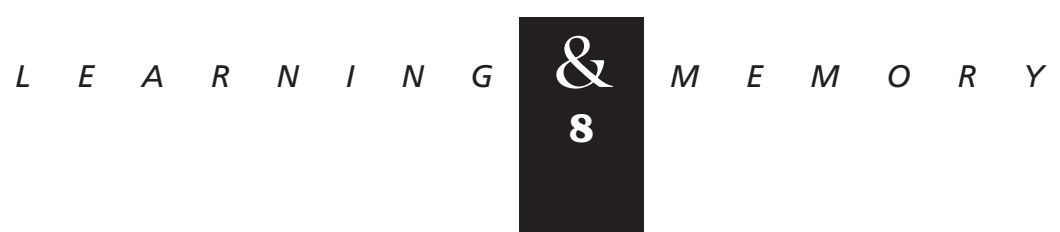


Table 3: Dynamics of memory performance in tests with mobile fertilized female

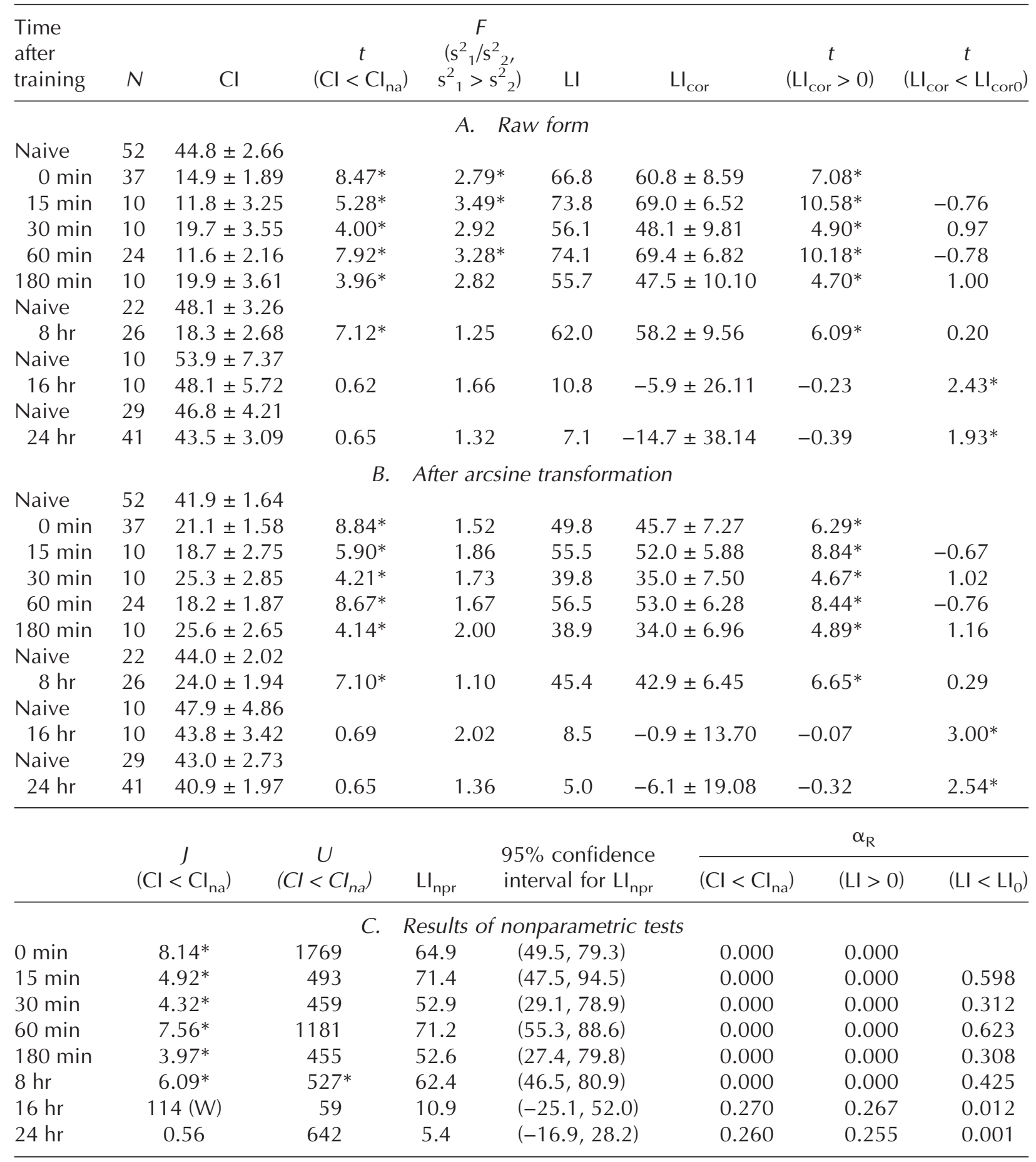

Memory performance was tested with mobile mated females instead of the usual test with immobilized virgin females. For each delayed test (8 or more hr after training), a separate sample of naive males was tested simultaneously with trained males. $W$ is the statistic of Wilcoxon. All other designations are as in Table 2.

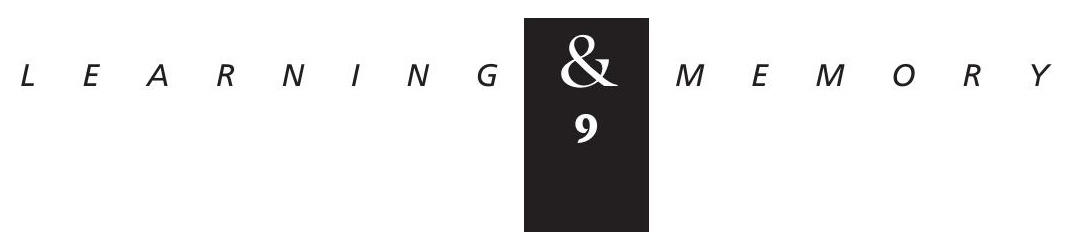


Kamyshev et al.

Table 4: Comparison of retention and retraining tests

\begin{tabular}{cccc}
\hline $\begin{array}{l}\text { Time after } \\
\text { training }(\min )\end{array}$ & $\begin{array}{c}t \\
\left(\mathrm{LI}_{\text {corM }} \neq \mathrm{LI}_{\text {corV }}\right)\end{array}$ & $\begin{array}{c}\alpha_{\mathrm{R}} \\
\left(\mathrm{LI}_{\mathrm{M}} \neq \mathrm{LI}_{\mathrm{V}}\right)\end{array}$ & $\begin{array}{c}\text { Overlapping of confidence } \\
\text { intervals for } \mathrm{LI}_{\text {npr }}\end{array}$ \\
\hline 0 & 0.04 & 0.514 & yes \\
15 & $3.41^{*}$ & 0.044 & no \\
30 & -0.18 & 0.588 & yes \\
60 & $3.80^{*}$ & 0.004 & no \\
180 & $2.64^{*}$ & 0.019 & yes \\
\hline
\end{tabular}

Results of two-tailed joint $t$ - and randomization tests for the null hypothesis that the LIs at a given time after training do not differ in tests with mated $\left(\mathrm{LI}_{M}\right.$ and $\left.\mathrm{LI}_{\text {corM }}\right)$ and virgin $\left(\mathrm{LI}_{\vee}\right.$ and $\left.\mathrm{LI}_{\text {corV }}\right)$ females. $\left.{ }^{*}\right)$ Differences significant at $\alpha=0.05$ according to the $t$-test. The fourth column is a summary of those cases where there is an overlap of $95 \%$ confidence intervals for $\mathrm{LI}_{\mathrm{npr}}$.

for $\mathrm{LI}_{\mathrm{cor}}$, the randomization test for LI, and overlapping of confidence intervals for $\mathrm{LI}_{\mathrm{npr}}$ (except the 3-hr point). Thus, testing males with mated females does not affect memory performance soon after training (see 0 - and 30-min points) but prevents memory lapse 1 and $3 \mathrm{hr}$ later, which is clearly observed in the test with virgins. The anomalous behavior of males in the test with virgins $15 \mathrm{~min}$ after training, which is also revealed by confidence intervals for $\mathrm{LI}_{\mathrm{npr}}$ in Table $2 \mathrm{C}$, will be discussed later. Table 4 is the last to compare different statistical approaches. Hereon, we shall use only randomization tests as the most correct and efficient method for comparing LIs (see Discussion).

IS SENSIBILIZATION TO THE ANTIAPHRODISIAC RESPONSIBLE FOR BETTER PERFORMANCE IN THE RETRAINING TEST?

The improvement of memory performance in the retraining test with mated females may be partly owing to nonassociative behavioral modifications such as an increase in sensitivity (sensibilization) to the antiaphrodisiac. This possible component of courtship reduction is obviously absent in the test with virgins. To rule out this possibility it is sufficient to show that the same degree of courtship reduction (as revealed by LI) occurs in the test with virgins that follows immediately after the retraining test, as in the retraining test itself.

The test with an immobilized virgin female was performed $3 \mathrm{hr}$ after training when memory performance is normally extremely reduced in comparison to the initial test just after training. A mated female was presented to an experimental male 2 hr 55 min after training for 5 min (retraining), immediately thereafter he was tested with a virgin female. This experimental variant, which includes both training and retraining $(T+R)$, is compared with two controls: training only $(\mathrm{T})$ and retraining pretest only (R). Memory performance in the group $\mathrm{T}$ was not significant $3 \mathrm{hr}$ after training (Table 5). Conversely, two other groups ( $\mathrm{T}+\mathrm{R}$ and

Table 5: Effect of 5-min retraining pretest on memory performance in tests with virgin females $3 \mathrm{hr}$ after training

\begin{tabular}{lrcccc}
\hline Group & $N$ & $\mathrm{Cl}$ & $\mathrm{LI}$ & $\begin{array}{c}\alpha_{\mathrm{R}} \\
\left(\mathrm{Cl}<\mathrm{Cl}_{\mathrm{na}}\right)\end{array}$ & $\begin{array}{c}\alpha_{R} \\
(\mathrm{LI}>0)\end{array}$ \\
\hline Naive & 9 & $80.5 \pm 4.6$ & & & \\
$\mathrm{~T}$ & 10 & $71.9 \pm 7.4$ & 10.7 & 0.189 & 0.190 \\
$\mathrm{R}$ & 10 & $32.8 \pm 7.8$ & 59.3 & 0.001 & 0.000 \\
$\mathrm{~T}+\mathrm{R}$ & 10 & $26.1 \pm 6.9$ & 67.6 & 0.000 & 0.000 \\
\hline
\end{tabular}

Cls were recorded in tests with immobilized virgin female in four experimental groups: in naive males; in trained males $3 \mathrm{hr}$ after training (training alone, $\mathrm{T}$ ); in trained males, which just before the test encountered a mated female in a 5-min pretest (training plus retraining, $\mathrm{T}+\mathrm{R}$ ); in previously untrained males with a 5-min pretest with a mated female just before the test ("retraining" alone, R). Other designations are as in Table 2.

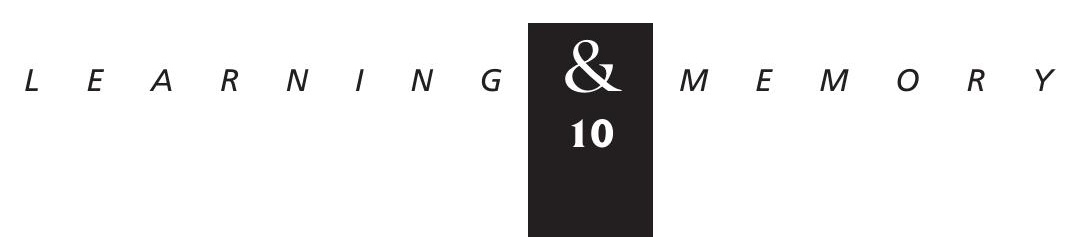


R) showed significant LIs. Both values are not significantly lower than LI values found in the test with mated females from $0 \mathrm{hr}$ to $8 \mathrm{hr}$ after training (cf. with the data from Table 3; one-tailed randomization test, $P>0.05$ ). We can thus conclude that (1) sensibilization to the antiaphrodisiac is not responsible for better performance in the retraining test and (2) essential conditioning may occur within the 5-min period (see group R). Consequently, the reconditioning may contribute to the performance revealed in the test with mated females.

\section{DOES LEARNING TO LEARN CONTRIBUTE}

TO THE TRANSFER OF TRAINING

IN CONDITIONED COURTSHIP?

One of the factors that may give the experimental subjects an advantage over the control subjects in the retraining test is known as "learning to learn," that is, the acquisition of learning skills as a result of practice (Postman 1971). In other words, in trained males additional acquisition during the retraining test may proceed faster than primary acquisition in naive males.

The dynamics of the CI reflecting the apparent learning in the course of 30-min training of naive males is presented in Figure 1. It is a rather slow process: a significant decline in CI in comparison with the first 5-min interval is observed only from 20 min after the start of training (one-tailed randomization test, $P<0.05)$.

Figure 2 presents the time course of the $\mathrm{CI}$ in naive and previously trained males during the test with mated females $8 \mathrm{hr}$ after training. The 5-min test period was subdivided into 20 successive 15 sec periods, and the data for the 8-hr point in Table 3 were recalculated. The rapid steady change in response to the mated female is observed in naive males during the transition from the first to the second 15-sec periods. The majority of the successive periods (including the final two) show CIs that are significantly lower than the CI for the first period (one-tailed randomization test, $P<0.05$ ). No significant decrease in the CI of naive males is observed in subsequent periods compared with the second period (one-tailed randomization test, $P>0.05)$. Thus, no apparent learning is observed within the first $5 \mathrm{~min}$ of training in naive males. The rapid initial decrease in CI probably reflects simple interruption of initially intensive courtship by the first presentation of the antiaphrodisiac or by the female's repelling movements (escape). No additional acquisition takes place in the previously trained flies during the 5-min test $8 \mathrm{hr}$ after training. CIs in all subsequent 15 -sec periods (except for the eighth) are not lower than the CI for the first period (one-tailed randomization test, $P>0.05$ ). For all periods, the CI of trained males is significantly lower than the CI of naive males (onetailed randomization test, $P<0.05$ ). From the very first seconds of the test, previously trained males demonstrate steady courtship reduction in comparison with naive males. This indicates that learning to learn does not contribute to the performance during the retraining test. The absence of a rapid response, which is observed in naive males, means that previously trained males avoid courtship from the very beginning of the test period. No escape response is observed.

\section{DO RESPONSES TO THE FEMALE'S BEHAVIOR CONTRIBUTE TO THE TRANSFER OF TRAINING IN CONDITIONED COURTSHIP?}

The retraining and retention tests are differed not only because of the pheromonal state of the females used, but also because in the retention test

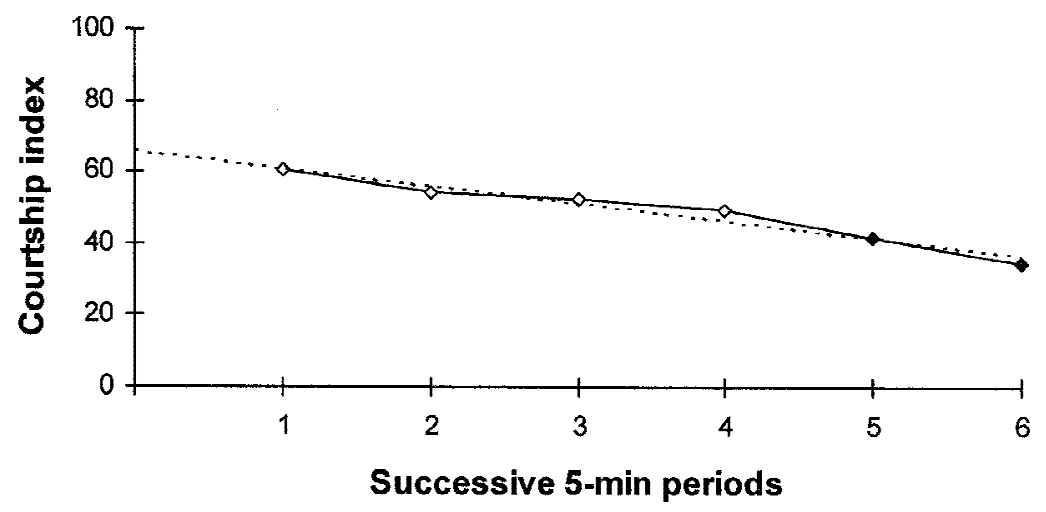

Figure 1: Time course of the mean $\mathrm{Cl}$ during 30-min training of naive males $(N=12)$. The symbols are solid when the one-tailed randomization test shows a significant $(P<0.05)$ decrease in $\mathrm{Cl}$ for a given period in comparison to the first 5 -min period. The linear trend line is shown.

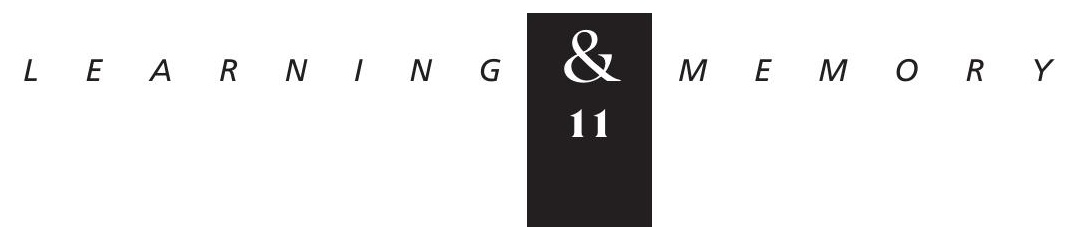


Figure 2: Time course of the mean $\mathrm{Cl}$ in naive $(\diamond, N=22)$ and previously trained males $(\square, N=26)$ in tests with mated female $8 \mathrm{hr}$ after training. For each curve, the symbols are solid when the one-tailed randomization test shows a significant $(P<0.05)$ decrease in $\mathrm{Cl}$ for a given period in comparison to the first 15-sec period. Linear trend lines are shown.

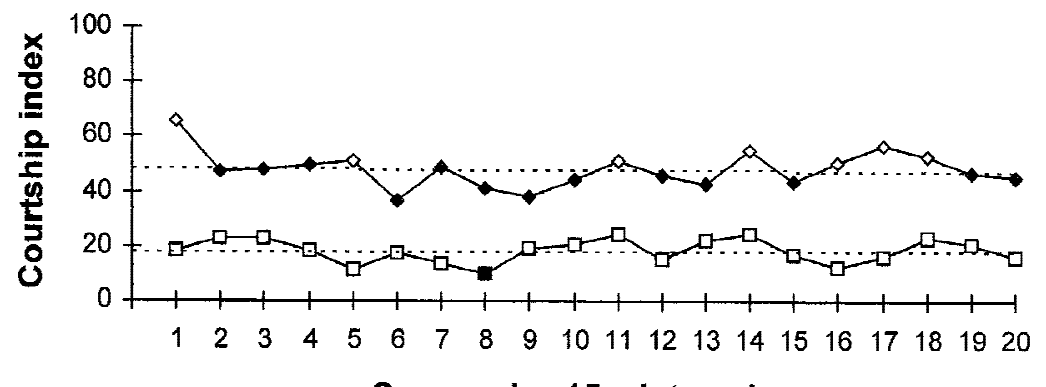

Successive 15-s intervals females are ether-immobilized. Thus, the transfer of training revealed in the test with mobile mated females may include factors based on the male's response to the female's behavior. To estimate this component, we compared memory performance in the tests with mobile and ether-immobilized mated females $3 \mathrm{hr}$ after training. Immobilized mated females were courted by another male just before the immobilization. Test with ether-immobilized virgins was included as a control. Results are presented in Table 6 . In both retraining tests highly significant LIs were found, whereas in the retention test, as usual, the LI value was low and nonsignificant. Responses to the female's behavior do not contribute to the transfer of training.

\section{DOES INSTRUMENTAL CONDITIONING} CONTRIBUTE TO MEMORY PERFORMANCE?

Instrumental conditioning is based on the formation of an association between the behavioral response of an animal and reinforcement (e.g., Schwartz and Reisberg 1991). Possible instrumental components in conditioned courtship may include two associations of this kind. The first is between male courtship behavior and its punishment by the female's rejection behavior. We showed above that this does not contribute to the transfer of training. The second component is a possible association between male courtship behavior and its punishment through the presentation of an antiaphrodisiac in the course of courtship.

It is known that in Drosopbila behavioral alterations resulting from instrumental conditioning are transient in the absence of reinforcement (e.g., Wustmann et al. 1996). If instrumental conditioning contributes to memory performance in the test with mated females, where reinforcement is present, this component should be rapidly extinguished in the absence of reinforcement. To see whether rapid extinction of conditioned courtship takes place in the retention test performed immediately after training, we recalculated the data in Table 2 for $0 \mathrm{hr}$ using the 15 -sec time intervals (Fig. 3 ). In case of extinction one should expect a positive trend in the CI of trained males and a gradual decrease in the difference for CI between naive and trained males. The dynamics of the $\mathrm{CI}$ in trained males showed a negative trend (Fig. 3A), whereas the dynamics of the difference score showed a slightly positive linear trend (Fig. 3B). No evidence was found that instrumental conditioning contributes to memory performance.

Table 6: Comparison of memory performance in tests with mobile mated females, ether-immobilized mated females, and ether-immobilized virgin females $3 \mathrm{hr}$ after training

\begin{tabular}{|c|c|c|c|c|c|}
\hline Female & Male & $N$ & $\mathrm{Cl}$ & $\mathrm{LI}$ & $\begin{array}{c}\alpha_{\mathrm{R}} \\
(\mathrm{LI}>0)\end{array}$ \\
\hline \multirow[t]{2}{*}{ Mobile mated } & naive & 18 & $53.9 \pm 7.1$ & & \\
\hline & trained & 20 & $20.8 \pm 4.0$ & 61.4 & 0.000 \\
\hline \multirow[t]{2}{*}{ Immobilized mated } & naive & 18 & $51.1 \pm 9.1$ & & \\
\hline & trained & 20 & $22.6 \pm 6.1$ & 55.8 & 0.008 \\
\hline \multirow[t]{2}{*}{ Immobilized virgin } & naive & 20 & $41.9 \pm 6.7$ & & \\
\hline & trained & 20 & $38.0 \pm 6.2$ & 9.3 & 0.330 \\
\hline
\end{tabular}

\begin{tabular}{lllllllllllllllll}
\hline & $A$ & $R$ & $N$ & $I$ & $N$ & $G$ & $\mathbf{Q}$ & $M$ & $E$ & $M$ & $O$ & $R$ & $Y$
\end{tabular}


A

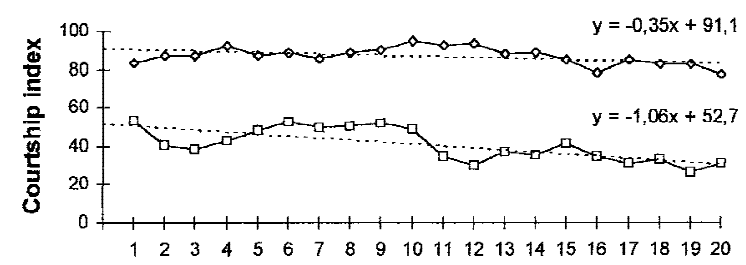

B

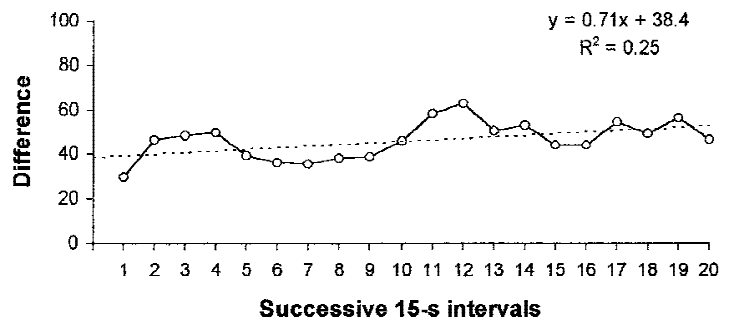

Figure 3: $(A)$ Time course of the mean $\mathrm{Cl}$ in naive $(\diamond$, $N=27)$ and trained males $(\square, N=27)$ in tests with a virgin female immediately after training. (B) Time course of the difference in $\mathrm{Cl}$ between naive and trained males. The linear trend lines and equations are shown. $R^{2}$ is the determination coefficient showing what part of the total variability is explained by the linear trend.

\section{Discussion}

\section{ESTIMATING MEMORY PERFORMANCE: THE VALIDITY OF VARIOUS TYPES OF STATISTICAL METHODS}

To compare learning ability or memory performance in experimental groups that differ for the courtship activity of control naive males, Gailey et al. (1982, 1984) introduced the LI (equation 2). Although objections have been made (see discussion in Gailey et al. 1991), there is no better way to measure the extent of conditioned suppression than to relate it to the initial courtship activity of naive males. The same difference score of 10 reflects a different extent of courtship reduction in the following two cases: $\mathrm{CI}_{\text {na }}=90$ versus $\mathrm{CI}_{\mathrm{tr}}=80$ and $\mathrm{CI}_{\mathrm{na}}=20$ versus $\mathrm{CI}_{\mathrm{tr}}=10$, the corresponding LIs being 11 and 50. Comparing the two LIs does, however, present a number of statistical problems.

Three different ways of dealing with conditioned courtship data, particularly for comparing two LIs, have been applied and compared here (see Materials and Methods): the approach based on the normal theory as described in van Swinderen and Hall (1995), the distribution-free approach based on Wilcoxon's statistic, and the dis- tribution-free approach based on the randomization test.

What are the limitations and efficiency of using these approaches in the simplest situation: testing $H_{0}: \mathrm{CI}=\mathrm{CI}_{\text {na }}$ against $H_{1}: \mathrm{CI}<\mathrm{CI}_{\text {na }}$ (Tables 2 and 3)? The arcsine transformation, which is customarily used for equalizing variances in the case of a binomial data distribution and which has been applied to conditioned courtship data by van Swinderen and Hall (1995), sometimes resulted in a sufficient decrease of $F$-ratio (Table 3 ), but this was not generally the case (Table 2). This transformation cannot be relied on to make sure that the $t$-test is valid. Instead, it is better to use nonparametric statistics, such as the Wilcoxon $W(J)$ and the Mann-Whitney $U$. However, these rank-based statistics are less efficient at revealing small differences than those based on interval scales (e.g., Runyon 1977; compare $t, J, U$, and $\alpha_{\mathrm{R}}$ statistics for 3-hr point in Table 2 ). The randomization test is both distribution free and sensitive, being based on an interval scale. Throughout this study, it leads to the same conclusions about significance of the difference $\mathrm{CI}_{\mathrm{na}}-\mathrm{CI}_{\mathrm{tr}}$ as the $t$-test.

The arcsine transformation of data results in a decrease of LI values that is more pronounced for high LI values (Table 3). Correction for bias only slightly affects the value of the LI for high and intermediate values. For low LI values this leads to a pronounced distortion of the data, making $\mathrm{LI}_{\text {cor }}$ sometimes even negative, whereas the difference $\mathrm{CI}_{\mathrm{na}}-\mathrm{CI}_{\mathrm{tr}}$ is still positive (Table 3 ). In contrast to an LI based on raw data, the distortions resulting from both the arcsine transformation and correction for bias make $\mathrm{LI}_{\text {cor }}$ a statistic that is difficult to interpret. The values of $t$ calculated to estimate the significance of $\mathrm{LI}_{\text {cor }}$ are quite different from those used to estimate the significance of the difference $\mathrm{CI}_{\text {na }}-\mathrm{CI}_{\mathrm{tr}}$. This leads to contradictory conclusions (Table 2) and is undoubtedly a reason for not using $\mathrm{LI}_{\text {cor. }}$ In contrast, conclusions made on the basis of LI used with the randomization test always agreed with those made for the difference score both by the $t$ - and randomization tests. As a rule, values of $\mathrm{LI}_{\mathrm{npr}}$ and $\mathrm{LI}$ are close $\mathrm{LI}_{\mathrm{npr}}$ is a median of all possible differences between individual scores of $\mathrm{CI}_{\text {na }}$ and $\mathrm{CI}_{\mathrm{tr}}$ ). Estimation of the significance of $\mathrm{LI}_{\mathrm{npr}}$ by means of the Moses' 95\% confidence interval completely agreed with the two-tailed Wilcoxon and Mann-Whitney tests.

The results of pairwise comparisons of LIs by means of $t$-test for $\mathrm{LI}_{\text {cor }}$ and the randomization test for LI also agreed (Tables 2-4). The utility of con-

$$
\begin{array}{llllllllllllllll}
L & E & A & R & N & I & N & G & \boldsymbol{Q} \\
\mathbf{1 3} & M & E & M & O & R & Y
\end{array}
$$


fidence intervals for pairwise comparisons of $\mathrm{LI}_{\mathrm{npr}}$ is limited by the fact that the partial overlap of confidence intervals can be seen for both significant and nonsignificant differences. Only some significant differences may be detected: when corresponding confidence intervals do not overlap. This test should therefore be considered a conservative one. However, we did find one example when the difference in $\mathrm{LI}_{\mathrm{npr}}$ should have been considered as significant, although neither $t$-, nor the randomization tests revealed a significant difference in the corresponding $\mathrm{LI}_{\mathrm{cor}}$ and LI (Table 2, difference between 15-min and 0-min points). This illustrates the fact that $\mathrm{LI}_{\mathrm{npr}}$ and LI reflect different qualities of the data structure.

An important conclusion flows from this comparison of the three different statistical approaches. When it is necessary to present conditioned courtship data as LIs, it is better to use randomization tests that allow LI to be analyzed directly. No transformations or corrections are necessary as with parametric analysis. The randomization test enables exact statistical comparisons to be performed, focusing on only those that are of real interest (in contrast to joint confidence intervals in ANOVA). Its advantages and applications to biological data are discussed in detail in Sokal and Rohlf (1995).

\section{SPECIFIC AND NONSPECIFIC COMPONENTS} OF TRANSFER IN CONDITIONED COURTSHIP

The fundamental principles of training transfer were discovered in experiments on verbal learning in humans. However, all sorts of animals display both positive and negative transfer effects, and the principles of transfer can be considered to apply across the animal kingdom (Houston 1981). The basic transfer experiment involves two stages. The subject first learns one task (quite often a pairedassociate list) and then attempts to master a second somewhat similar task. If the stimuli in the two successive lists are identical but the responses in the two successive lists are different, this is called an A-B, A-C paradigm. If the stimuli are different but the responses are identical, this is called an A-B, C-B paradigm, etc. The situation when both the stimuli and the responses are identical in the two successive tasks, as in our case, is referred to as an A-B, A-B paradigm.

Nonspecific transfer results from such factors as learning to learn and warm-up (Houston 1981). These factors are independent of the specific con- tents of the lists. When subjects show improvement in their ability to learn the successive tasks, this is learning to learn. We have seen that in the case of conditioned courtship, learning to learn does not contribute to memory performance during the 5-min retraining test (Fig. 2). Warm-up may be defined as the establishment of a transitory set of activities that prepares the subject for the perceptual and motor requirements of the experiment (Postman 1971). To facilitate the subsequent performance of the learning task, warm-up activity need not involve any learning. It may include the simple adaptation of the subject to the experimental situation and the requirements of the learning task. There is a rapid dissipation of warm-up effects after the cessation of practice. Warm-up may contribute to the difference between naive and trained males (both in the retraining and retention tests) immediately after training but could hardly influence memory performance in delayed tests. One of the possible controls for warm-up-sham training-was performed (Table 1), but it showed no significant difference between naive and "warmedup" males either in the immediate retraining test or in the immediate retention test.

Transfer effects are described as specific when they are attributable to known similar relations between the components of successive tasks (Postman 1971). To identify the relevant components of successive tasks, the stimulus-response analysis is used. In an early study, Poffenberger (1915) proposed three generalizations: Transfer is positive in the A-B, A-B paradigm, negative in the A-B, A-C paradigm, and zero in the A-B, C-D paradigm. Wylie (1919) in a study of maze learning with rats added a fourth principle: Transfer effects are positive when the stimulus changes and the response remains the same (A-B, C-B). Although the relationship between transfer and intertask similarity is much more complex (see Postman 1971; Houston 1981), we shall ignore these considerations, because only the simplest transfer paradigm (A-B, A-B) applies here. The main component of specific transfer in the present case seems to be the association between $\mathrm{A}$ and $\mathrm{B}$, that is, aphrodisiac-antiaphrodisiac, or CS-US, association. However, transfer is not a single, simple process. Learning a simple verbal association involves at least four subprocesses: stimulus learning, response learning, forward stimulus-response association, and backward response-stimulus association (Houston 1981). What are the possible components of specific transfer in conditioned courtship? To answer

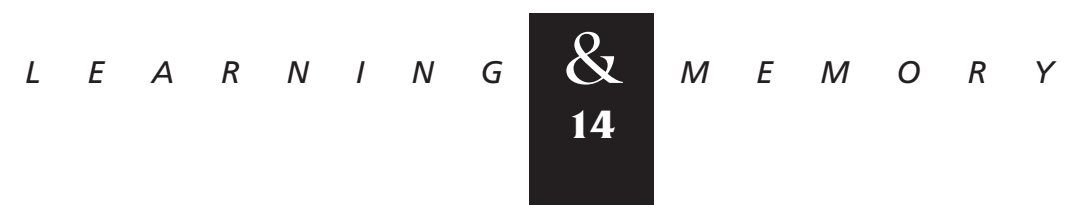


this question, we need a working model of what is learned during the training of the male with a mated female.

\section{A WORKING MODEL FOR CONDITIONED COURTSHIP SUPPRESSION IN DROSOPHILA}

Zawistowski (1988) was the first to point out that conditioned courtship suppression in Drosopbila results from the counterconditioning of attractive and aversive stimuli. Counterconditioning was first described by Erofeeva $(1916,1921)$ who reported from Pavlov's laboratory that a strong salivary response, reinforced by food, could be conditioned to a painful electric shock as a CS in dogs. Here, we use a model of counterconditioning developed by Dickinson and Dearing (1979) on the basis of Konorski's general view of conditioning and motivational processes.

Konorski (1967) distinguished between two systems that mediated properties of the US. Presenting the US first activates some form of its internal sensory representation. This, in turn, arouses a response mechanism that determines the actual form of the "consummatory" reflex, such as salivation or leg flexion, seen in response to that particular US. This system mediates the sensory properties of the stimulus. The activation of an internal representation of a stimulus with "emotional" significance will also lead to the arousal of the central motivational system, which mediates the general emotive and reinforcing properties of the stimulus. Arousal of the motivational system (1) feeds back on the sensory representation of the stimulus to potentiate the consummatory response and (2) leads to the emission of "preparatory" behavior, such as approach or withdrawal, that reflects the general affective character (attractive or aversive) of the stimulus.

Konorski (1967) thought that when a neutral CS is paired with a US in a classical conditioning paradigm, the internal representation of the CS becomes connected by independent excitatory associations with both the representation of the US and the motivational system, with the reinforcement process depending on the arousal of the motivational system by the US.

These ideas, as applied to counterconditioning by Dickinson and Dearing (1979), are presented in Figure $4 \mathrm{~A}$. We adapted their scheme to the counterconditioning of an attractive US, the aphrodisiac. Pairings with the aversive US, the antiaphrodisiac, in the course of training with a mated fe- male transform the attractive US, the aphrodisiac, into an aversive CS. Two conditioned connections are established. The first is a direct association between the CS and the aversive motivational system. The second association is formed between internal representations of the CS and the US. The US representation has a pre-established excitatory connection to the aversive system. Combined arousal of the aversive system by the two excitatory connections leads to the inhibition of the appetitive motivational system that diminishes the unconditioned response to the aphrodisiac (courtship suppression).

Dickinson and Dearing (1979) emphasize that the unconditioned response to a stimulus could only be changed by counterconditioning if the elicitation of that response involves motivational processes. Moreover, changes in the affective properties of the stimulus subject to counterconditioning occur in the absence of changes in the consummatory response actually elicited by that stimulus. Thus, the central units in the mechanism of counterconditioning are the aversive and appetitive motivational systems with their reciprocal inhibitory interactions.

Dickinson and Dearing (1979), using Wagner's model of associative conditioning, also point out how the CS-US connection may work. According to Wagner (Wagner 1979, 1981), the development of an association depends not just on the pairing of the CS and the US but on their active, simultaneous processing. The association between the CS and the US will be formed only to the extent that they are rehearsed together. Once the association is formed, presentation of the CS alone retrieves a representation of the US from some long-term store into a limited-capacity rehearsal mechanism. The presence of this representation in the rehearsal mechanism controls the conditioned response. There is no reason why the retrieved representation should contain all the information encoded in a representation of the same US that originates from the actual presentation of that US (Dickinson and Dearing 1979). That is why representations of the US elicited by the actual US and by a CS are presented separately in Figure $4 \mathrm{~A}$.

What we need to explain first using this model is the difference in the dynamics of memory performance seen in the retention and in the retraining tests. The main connections leading to conditioned courtship suppression in each case are shown in Figure 4, B and C. Memory performance in the test with a virgin female (in absence of the

$$
\begin{array}{lllllllllllllll} 
& E & A & R & N & I & N & G & \begin{array}{l}
\boldsymbol{Q} \\
15
\end{array} & M & E & M & O & R & Y
\end{array}
$$




\section{Kamyshev et al.}

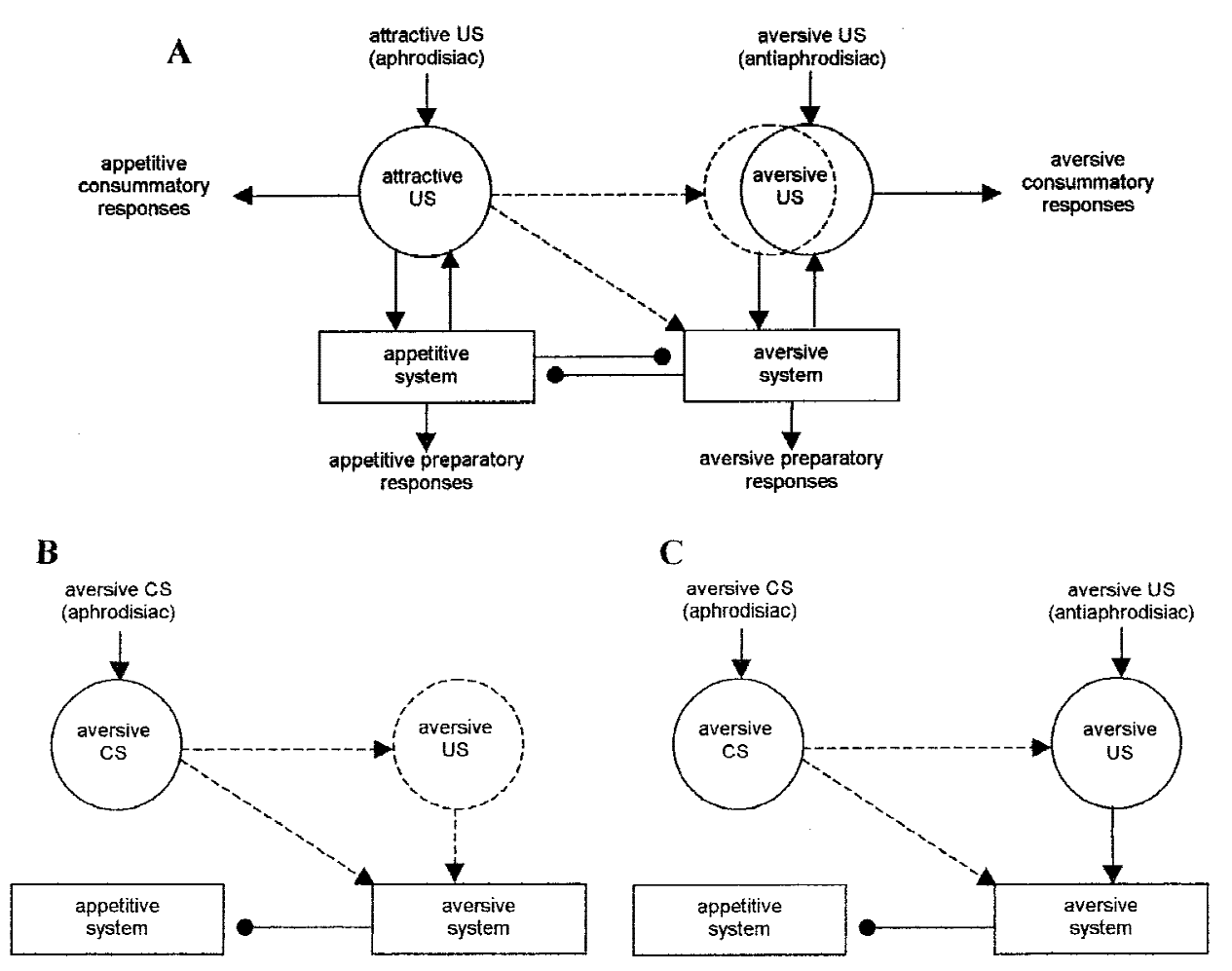

Figure 4: (A) Counterconditioning of the aphrodisiac as a result of its pairings with the antiaphrodisiac (adapted from Dickinson and Dearing 1979). ( $\longrightarrow$ ) Preestablished excitatory connections; $(\longrightarrow)$ preestablished inhibitory connections; $(-\rightarrow)$ excitatory connections established by conditioning; circles indicate internal sensory representation of a stimulus excited by either unconditioned (solid circles) or conditioned (broken line circle) connections. (B) Main connections involved in the inhibition of the appetitive motivational system during the presentation of the aphrodisiac in the retention test. The strength of the US input to the aversive system depends on the retention of an effective internal representation of the US and the retention of the CS-US association. (C) Main connections involved in the inhibition of the appetitive motivational system during the presentation of the aphrodisiac in the retraining test. The presentation of the US in the test re-establishes its effective internal representation and provides its normal excitatory input to the aversive system. During presentation of the aphrodisiac, the extent of arousal of the aversive system from the US depends only on the retention of the CS-US association.

US) reflects retention of three different memories related to (1) the association between the CS and the aversive system, (2) the association between internal representations of the CS and US, and (3) the internal representation of the US. The latter may change with time, finally being substantially different from the representation that originates from the actual presentation of the antiaphrodisiac. This leads to the weakening of the excitatory connection between internal representation of the US and the aversive system. Activation of the aversive system by the CS-US association therefore depends here on the retention of a proper representation of the US. Memory lapse is observed $3 \mathrm{hr}$ after training (Table 2), when both the CS-US and the CS-aversive system associations no longer activate the aversive system.
In the retraining test with a mated female, a proper internal representation of the US is re-established owing to its presentation in the test. The length of the period for which the positive transfer is observed (at least $8 \mathrm{hr}$ after training; see Table 3) is limited by the retention of the CS-US association.

The model can also explain the recent results of Kane et al. (1997). In their experiments, transgenic flies specifically inhibited for protein kinase $\mathrm{C}$ showed no courtship reduction during a 1-hr training period with mobile fertilized females but demonstrated normal memory in tests with immobilized virgin or mated females 10 min after training. They called this phenomenon "learning without performance." A common stimulus-a mated female-was supposed to trigger separate events: a

$$
\begin{array}{lllllllllllllll}
L & E & A & R & N & I & N & G & \begin{array}{l}
\boldsymbol{Z} \\
16
\end{array} & M & E & M & O & R & Y
\end{array}
$$


protein kinase C-dependent process that feeds back immediately on the animal's behavior and one or more separate processes that lead to memory formation. Our results also show learning without performance, but in normal flies. On the one hand, the 5-min training period is not sufficient to produce any visible changes in courtship intensity (Fig. 2, naive males, 2nd-20th periods). On the other hand, it is sufficient to produce courtship reduction in a further retention test (cf. groups $R$ and naive in Table 5). This supports the hypothesis that performance in the course of training and performance in the retention test are based on different processes. Classical conditioning based on an aphrodisiac-antiaphrodisiac (CS-US) association appears not to produce performance until cessation of the continuous training period. This may be important for aspects of the conditioning mechanism such as calculating the contingency ${ }^{3}$ between the US and the CS, one of the prerequisites for Pavlovian conditioning (e.g., Schwartz and Reisberg 1991). The component of learning that results in performance during training in normal flies and that is impaired in transgenic flies inhibited for protein kinase C (Kane et al. 1997) may be the formation of the CS-aversive system association. We suggest that the mechanism of formation of this association is somewhat different from the mechanism of formation of the CS-US association and, possibly, does not include calculation of contingencies.

What else may be included in the model? When a male courts a mated female, there is a contingency between male courtship behavior on the one hand and the presentation of the antiaphrodisiac or the female's rejection behavior on the other. In addition to the associations relevant to classical conditioning (see above), the responsereinforcement association, which is critical in instrumental conditioning (e.g., Schwartz and Reisberg 1991), should also be formed. Boakes (1979) points out that almost any situation is likely to contain, explicitly or implicitly, both stimulus-reinforcer and response-reinforcer contingencies, and the range of conditions under which classical-instrumental interactions occur is very large. The term "classical-instrumental interaction" refers to a variety of phenomena that have only one thing in common: They involve two separate conditioning processes (classical and instrumental), which compete, complement, or otherwise interact with each other (Boakes 1979). For example, classical conditioning may block or overshadow instrumental conditioning, and vice versa. The role of an instrumental component in conditioned courtship has been pointed out previously (e.g., Zawistowski 1988; Gailey and Siegel 1989). However, the nature of the classical-instrumental interaction in this form of learning is unknown. For that reason, we have not included it in the present model.

\section{COMPONENTS OF SPECIFIC TRANSFER IN CONDITIONED COURTSHIP}

According to the model presented above, two main components of the specific transfer in conditioned courtship appear to be the association between the CS and the aversive system and the association between internal representations of the CS and US. The first component has a shorter retention than the second and does not contribute to transfer in delayed tests ( $3 \mathrm{hr}$ and later).

The components of specific transfer may also include nonassociative behavioral modifications. There are two possible nonassociative effects that may contribute to the courtship reduction observed in the retraining test. These are habituation to the aphrodisiac and an increase in sensitivity (sensibilization) to the antiaphrodisiac. It was shown that the former has little or no significance for conditioned courtship suppression (Ackerman and Siegel 1986; Zawistowski 1988). Here, we have shown that sensibilization to the antiaphrodisiac does not contribute significantly to training transfer, because the same degree of courtship reduction is seen in the test with virgins (i.e., in absence of the direct action of the antiaphrodisiac) immediately following the retraining test and in the retraining test itself (Table 5).

The possible association between male courtship behavior (response) and its punishment by the female's rejection behavior (reinforcement) does not contribute significantly to the transfer of

\footnotetext{
${ }^{3}$ Temporal contiguity of the CS and the US (in the case of Pavlovian conditioning) or of response and reinforcement (in the case of instrumental conditioning) is not sufficient for conditioning to occur. There must also be a differential contigency between them. This means that the probability of the paired presentation of the US and the CS should be different from the probability of presenting the US in the absence of the CS. In other words, organisms are sensitve to the extent to which one stimulus provides information about the other. Similarly, they can distinguish response-dependent from response-independent reinforcement. See Schartz and Reisberg (1991) for a full explanation.
}

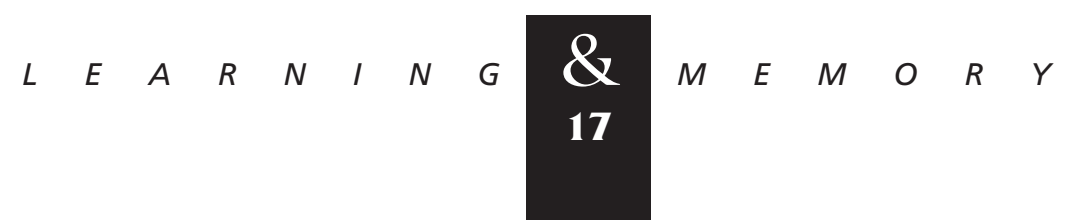


training. This follows from two facts: (1) In Drosopbila the behavioral alterations resulting from operant conditioning are very transient in the absence of reinforcement (e.g., Wustmann et al. 1996), and (2) memory performance $3 \mathrm{hr}$ after training is similar with mobile and immobilized mated females (Table 6). More generally, we find no support for the role of instrumental conditioning of any type to the memory performance in conditioned courtship. Rapid extinction, expected with instrumental conditioning, was not found in the immediate retention test (Fig. 3).

It seems most likely that the two associative connections described in our model are the only factors involved in transfer in conditioned courtship.

\section{TESTS WITH MATED FEMALE AS A NEW PROTOCOL IN CONDITIONED COURTSHIP}

The retraining test can be seen as a new protocol for testing conditioned courtship in addition to the commonly used retention test. In the retention test with a virgin female, memory retention is limited either by the retention of the direct association between the CS and the aversive system or by the retention of a proper internal representation of the US. In the retraining test, memory performance is limited by the retention of the CS-US association. Three or more hours after training, when no memory is detectable in the retention test, this association seems to be the only factor involved in transfer. For details of the protocol, see Materials and Methods.

Why use mobile mated females instead of ether-immobilized females? (1) In the relatively large chambers we use, a male is more rapidly stimulated to court by a mobile female rather than by an immobilized individual. (2) A mobile mated female is always ready to respond to male courtship by presenting the antiaphrodisiac, whereas in the test with an immobilized female, elicitation of the antiaphrodisiac is induced by another male courting her just before immobilization (Gailey et al. 1984; Gailey and Siegel 1989). (3) Presentation of a mobile mated female in the test reproduces the training situation more accurately. (4) The experimenter can regulate the observation time irrespective of the duration of ether narcosis. A long observation time may be required to avoid a "floor" effect, that is, an inability to observe experiencedependent courtship reduction owing to a low level of courtship in naive males.
THE DYNAMICS OF MEMORY PERFORMANCE IN CONDITIONED COURTSHIP

The difference in memory dynamics observed in the retention and retraining tests has already been explained using our model. However, one aspect remains to be discussed: the reduction in memory performance in the test with a virgin female 15 min after training, which is partially restored $30 \mathrm{~min}$ after training. Because of this effect, the difference between the LIs in the retention and retraining tests is nonsignificant at 0 - and 30-min points but significant at the 15 -min point (Table 4 ). In mammals the transient deterioration of memory performance soon after training, followed by a period of spontaneous improvement and then by long-term forgetting, is known as the Kamin effect (Kamin 1957) and seems to result from a failure in the retrieval process. This is shown by the fact that different retrieval cues may reactivate the memory trace (Gisquet-Verrier et al. 1989). In our case, this negative wave in the memory retention may derive from a transient failure in the retrieval of the internal US representation. This has no consequence on memory performance in the retraining test, where US representation is re-established in the rehearsal mechanism owing to its presentation.

It is interesting to compare the dynamics of memory performance revealed in the retraining test with memory retention in other classical conditioning paradigms in Drosopbila. Using the odor-shock paradigm, Tully et al. (1994) have shown that the memory retention curve depends on the training mode. The memory after one cycle or massed training decays within 4 days. Spaced training produces robust memory retention that lasts at least 7 days. However, memories produced by all training modes show a phase of fast decay within the first hour after training that is obviously related to decay in short-term memory. Interestingly, we did not observe any significant decay in memory performance in the retraining test for $8 \mathrm{hr}$ after training. This is more like memory retention after positively reinforced conditioning. Tempel et al. (1983) observed stable memory performance after sucrose-reinforced conditioning for $3 \mathrm{hr}$ after training.

Unlike Gailey et al. (1984), who found that courting a fertilized female suppresses courtship of fertilized females for nearly 1 day, we found no memory $16 \mathrm{hr}$ after training. However, if courtship is important for mating success (for an opposing view, see Zawistowski and Richmond 1985, 1987),

$$
\begin{array}{lllllllllllllll}
L & E & A & R & N & I & N & G & \begin{array}{l}
\boldsymbol{Z} \\
\mathbf{1 8}
\end{array} & M & E & M & O & R & Y
\end{array}
$$


long-lasting courtship inhibition may lead to a decrease in evolutionary fitness.

\section{Acknowledgments}

This work was supported by a grant from the Russian Foundation of Basic Research (project code 96-04-00099G) and partially by the Russian Research Program "Paramount Directions in Genetics." We thank Dr. Elena Savvateeva for her help in the preparation of the manuscript. We are grateful to the anonymous referees for their comments. We are especially grateful to Dr. Matthew Cobb for final corrections to the manuscript.

The publication costs of this article were defrayed in part by payment of page charges. This article must therefore be hereby marked "advertisement" in accordance with 18 USC section 1734 solely to indicate this fact.

\section{References}

Ackerman, S.L. and R.W. Siegel. 1986. Chemically reinforced conditioned courtship in Drosophila: Responses of wild type and the dunce, amnesiac and don giovanni mutants. J. Neurogenet. 3: 111-123.

Bastock, M. and A. Manning. 1955. The courtship of Drosophila melanogaster. Behavior 8: 85-111.

Basu, D. 1980. Randomization analysis of experimental data: The Fisher randomization test. J. Amer. Stat. Assoc. 75: 575-595.

Boakes, R.A. 1979. Interactions between type I and type II processes involving positive reinforcement. In Mechanisms of learning and motivation. A memorial volume to Jerzy Konorski (ed. A. Dickinson and R.A. Boakes), pp. 233-268. Erlbaum, Hillsdale, NJ.

Connolly, K. and R. Cook. 1973. Rejection responses by female Drosophila melanogaster: Their ontogeny, causality, and effects upon the behavior of the courting male. Behaviour 52: 155-171.

Cook, R. and A. Cook. 1975. The attractiveness to males of female Drosophila melanogaster: Effects of mating, age, and diet. Anim. Behav. 23: 521-526.

Davis, R.L. 1996. Physiology and biochemistry of Drosophila learning mutants. Physiol. Rev. 76: 299-317.

DeZazzo, J. and T. Tully. 1995. Dissection of memory formation: From behavioral pharmacology to molecular genetics. Trends Neurosci. 18: 212- 218.

Dickinson, A. and M.F. Dearing. 1979. Appetitive-aversive interactions and inhibitory processes. In Mechanisms of learning and motivation. A memorial volume to Jerzy Konorski (ed. A. Dickinson and R.A. Boakes), pp. 203-231. Erlbaum, Hillsdale, NJ.

Dudai, Y. 1988. Neurogenetic dissection of learning and short-term memory in Drosophila. Annu. Rev. Neurosci. 11: 537-563.
Edgington, E.S. 1980. Randomization tests. Marcel Dekker, New York, NY.

Erofeeva, M.N. 1916. Contributions a l'etude des reflexes conditionnels destructifs. Compte Rendu de la Societé de Biologie Paris 79: 239-240.

1921. Additional data on nocuous conditioned reflexes. Izvestiya Petrogradskogo Nauchnogo Instituta im. P.F. Lesgafta 3: 69-73 (in Russian).

Ferveur, J.-F. 1997. The pheromonal role of cuticular hydrocarbons in Drosophila melanogaster. BioEssays 19: $353-358$.

Ferveur, J.-F., M. Cobb, and J.-M. Jallon. 1989. Complex chemical messages in Drosophila. In Neurobiology of sensory systems (ed. R.N. Singh and N.J. Strausfeld), pp. 397-409. Plenum Publishing Co., New York, NY.

Fisher, R.A. 1966. The design of experiments, 8th ed. Oliver and Boyd, Edinburgh, Scotland

Gailey, D.A. and R.W. Siegel. 1989. A mutant strain in Drosophila melanogaster that is defective in courtship behavioural cues. Anim. Behav. 38: 163-169.

Gailey, D.A., F.R. Jackson, and R.W. Siegel. 1982. Male courtship in Drosophila: The conditioned response to immature males and its genetic control. Genetics 102: $771-782$.

1984. Conditioning mutations in Drosophila melanogaster affect an experience-dependent behavioral modification in courting males. Genetics 106: 613-623.

Gailey, D.A., A. Villella, and T. Tully. 1991. Reassessment of the effect of biological rhythm mutations on learning in Drosophila melanogaster. J. Comp. Physiol. A 169: 685-697.

Gisquet-Verrier, P., A. Dekeyne, and T. Alexinsky. 1989. Differential effects of several retrieval cues over time: Evidence for time-dependent reorganization of memory. Anim. Learn. Behav. 17: 394-408.

Hall, J.C. 1994. The mating of a fly. Science 264: 1702-1714.

Heisenberg, M. 1989. Genetic approach to learning and memory (mnemogenetics) in Drosophila melanogaster. In Fundamentals of memory formation: Neuronal plasticity and brain function (ed. H. Rahmann), pp. 3-45. Gustav Fischer Verlag, Stuttgart, Germany.

Hollander, M. and D.A. Wolfe. 1973. Nonparametric statistical methods. John Wiley and Sons, New York, NY.

Houston, J.P. 1981. Fundamentals of learning and memory, 2nd ed. Academic Press, New York, NY.

Iman, R.L. 1976. An approximation to the exact distribution of the Wilcoxon-Mann-Whitney rank sum test statistic. Commun. Statist. A: Theory Method A5: 587-598. 


\section{Kamyshev et al.}

Kamin, L.J. 1957. The retention of an incompletely learned avoidance response. J. Comp. Physiol. Psychol. 50: 457-460.

Kane, N.S., A. Robichon, J.A. Dickinson, and R.J. Greenspan. 1997. Learning without performance in PKC-deficient Drosophila. Neuron 18: 307-314.

Konorski, J. 1967. Integrative activity of the brain: An interdisciplinary approach. University of Chicago Press, Chicago, IL.

Mackintosh, N.J. 1974. The psychology of animal learning. Academic Press, New York, NY.

Poffenberger, A.T. 1915. The influence of improvement in one simple mental process upon other related processes. J. Educat. Psychol. 6: 459-474.

Postman, L. 1971. Transfer, interference and forgetting. In Woodworth and Schlosberg's experimental psychology, 3rd ed. (ed. J.W. Kling and L.A. Riggs), pp. 1019-1132. Holt, Rinehart and Winston, New York, NY.

Quinn W.G., W.A. Harris, and S. Benzer. 1974. Conditioned behavior in Drosophila melanogaster. Proc. Natl. Acad. Sci. 71: 708-712.

Runyon, R.R. 1977. Nonparametric statistics. A contemporary approach. Addison-Wesley Publishing Co., Reading, MA.

Schwartz, B. and D. Reisberg. 1991. Learning and Memory. W.W. Norton \& Co., New York and London.

Siegel, R.W. and J.C. Hall. 1979. Conditioned responses in courtship behavior of normal and mutant Drosophila. Proc. Natl. Acad. Sci. 76: 3430-3434.

Sokal, R.R. and F.J. Rohlf. 1995. Biometry, 3rd ed. W.H. Freeman \& Co., New York, NY.

Spear, N.E. 1971. Forgetting as retrieval failure. In Animal memory (ed. W.K. Honig and P.H.R. James), pp. 47-109. Academic Press, New York, NY.

Tempel, B.L., N. Bonini, D.R. Dawson, and W.G. Quinn. 1983. Reward learning in normal and mutant Drosophila. Proc. Natl. Acad. Sci. 80: 1482-1486.

Tompkins L., R.W. Siegel, D.A. Gailey, and J.C. Hall. 1983. Conditioned courtship in Drosophila and its mediation by association of chemical cues. Behav. Genet. 13: 565-578.

Tully, T. and W.G. Quinn. 1985. Classical conditioning and retention in normal and mutant Drosophila melanogaster. J. Comp. Physiol. A 157: 263-277.

Tully, T., T. Preat, S.C. Boynton, and M. Del Vecchio. 1994. Genetic dissection of consolidated memory in Drosophila. Cell 79: 35-47.

van Swinderen, B. and J.C. Hall. 1995. Analysis of conditioned courtship in dusky-Andante rhythm mutants of Drosophila. Learn. \& Mem. 2: 49-61.
Wagner, A.R. 1979. Habituation and memory. In Mechanisms of learning and motivation. A memorial volume to Jerzy Konorski (ed. A. Dickinson and R.A. Boakes), pp. 53-82. Erlbaum, Hillsdale, NJ.

1981. SOP: A model of automatic memory processing in animal behavior. In Information processing in animals: Memory mechanisms (ed. N.E. Spear and R.R. Miller). Erlbaum, Hillsdale, NJ.

Wustmann, G., K. Rein, R. Wolf, and M. Heisenberg. 1996. A new paradigm for operant conditioning of Drosophila melanogaster. J. Comp. Physiol. A 179: 429-436.

Wylie, H.H. 1919. An experimental study of transfer of response in the white rat. Behav. Monographs, No. 16.

Zawistowski, S. 1988. A replication demonstrating reduced courtship of Drosophila melanogaster by associative learning. J. Comp. Psychol. 102: 174-176.

Zawistowski, S. and R.C. Richmond. 1985.

Experience-mediated courtship reduction and competition for mates by male Drosophila melanogaster. Behav. Genet. 15: $561-569$.

1987. Experience-mediated reduction in courtship of Drosophila melanogaster in large and small chambers. J. Comp. Physiol. 101: 90-93.

Received March 10, 1997; accepted in revised form November 4, 1998.

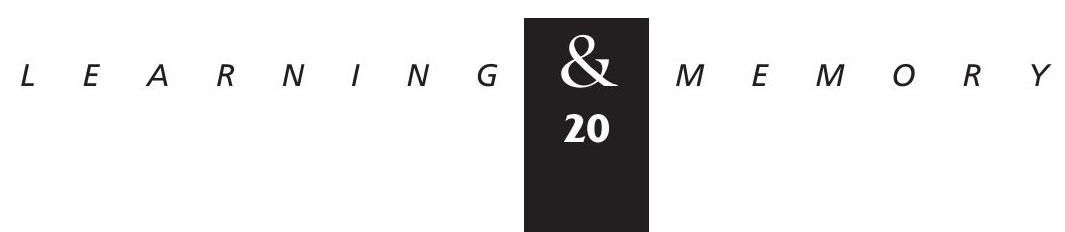




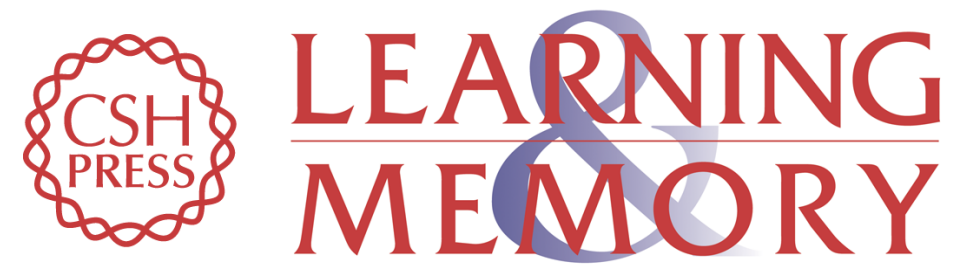

\section{Drosophila Conditioned Courtship: Two Ways of Testing Memory}

Nikolai G. Kamyshev, Konstantin G. Iliadi and Julia V. Bragina

Learn. Mem. 1999, 6:

Access the most recent version at doi:10.1101/lm.6.1.1

References This article cites 26 articles, 7 of which can be accessed free at: http://learnmem.cshlp.org/content/6/1/1.full.html\#ref-list-1

License

Email Alerting Receive free email alerts when new articles cite this article - sign up in the box at the Service top right corner of the article or click here. 